\title{
THE PLANAR SCHÖNFLIES THEOREM FOR LIPSCHITZ MAPS
}

\author{
PEKKA TUKIA
}

\section{Introduction}

1. Let $X$ and $Y$ be metric spaces with metrics $d$ and $d^{\prime}$. Let $f: X \rightarrow Y$ be an embedding such that for some $L \geqq 1$

$$
d(x, y) / L \leqq d^{\prime}(f(x), f(y)) \leqq L d(x, y)
$$

for all $x, y \in X$. Then we say that $f$ is a Lipschitz embedding, or, if we wish to emphasize that $f$ satisfies (1) with a particular number $L$, we say that $f$ is an $L$-embedding. If $f$ is a homeomorphism, we say that $f$ is a Lipschitz homeomorphism or, respectively, an $L$-homeomorphism, $L$ as in (1). If a map satisfies only the right side inequality of (1), we say that $f$ is L-Lipschitz.

We denote by $I$ the closed interval $[0,1]$, by $I^{1}$ the interval $[-1,1]$ and by $I^{2}$ the square $I^{1} \times I^{1}$. The boundary $\partial I^{2}$ is denoted by $S$. The metric of $R^{2}$ is the usual norm metric.

2. We will show that a Lipschitz embedding of $S$ or $I^{1}$ into $R^{2}$ can always be extended to a Lipschitz homeomorphism of $R^{2}$.

Theorem A (the Schönflies theorem for Lipschitz maps). Every L-embedding of the circle $S$ into $R^{2}$ can be extended to a $L^{\prime}$-homeomorphism of $R^{2}$, which is piecewise linear outside $S$ and where $L^{\prime}$ depends only on $L$.

Theorem B. Every L-embedding of $I^{1}$ into $R^{2}$ can be extended to a $L^{\prime \prime}$-homeomorphism of $R^{2}$, which is piecewise linear outside $I^{1}$ and where $L^{\prime \prime}$ depends only on $L$.

Theorem A should not be confused with the generalized Schönflies theorem, which assumes that the image is collared. In contrast to Theorem A (for a counterexample see $[6,3.10]$ ), the generalized Schönflies theorem is true in many categories for every $n$; in particular, it is true in the Lipschitz category (Luukkainen-Väisälä [6, Theorem 7.7]).

Using the generalized Schönflies theorem for Lipschitz maps one could prove a version of Theorem A by aid of Theorem B as follows: Let $f: S \rightarrow R^{2}$ be a Lipschitz embedding. Then, by Theorem B, $f(S)$ is Lipschitz collared locally. It follows 
[6, Theorem 7.4] that $f(S)$ has a global Lipschitz collar. Now, using the generalized Schönflies theorem, we could extend $f$ to $I^{2}$ to obtain a Lipschitz embedding $F$ of $I^{2}$ into $R^{2}$. However, it seems difficult to prove that $F$ is an $L^{\prime}$-embedding, where $L^{\prime}$ depends only on $L$. Therefore we give parallel proofs of Theorems A and $\mathrm{B}$; we give a detailed proof for Theorem $\mathrm{A}$ and then indicate the modifications needed for Theorem B.

3. Theorems A and B have the following corollary. Let $J \subset R^{2}$ be a rectifiable Jordan curve or arc. Let $d$ be the metric of $J$ for which $d(x, y)$ is the length of the subarc of $J$ with endpoints $x$ and $y$ (if there are two such arcs we let $d(x, y)$ be the length of the shorter arc). We say that $J$ is a Lipschitz curve or arc if id: $(J, d) \rightarrow$ $(J,\|\|)$ is a Lipschitz homeomorphism. If id is an $L$-homeomorphism we say that $J$ is an $L$-curve or an $L$-arc.

Corollary. A subset $J$ or $R^{2}$ is a Lipschitz curve or arc if and only if $J$ is the image of the circle $(\lambda / 8) S$ or the interval $(\lambda / 2) I^{1}$, where $\lambda$ is the length of $J$, under a Lipschitz homeomorphism $F$ of $R^{2}$. In addition, if $J$ is an L-curve or an L-arc, we may assume that $F$ is a $K$-homeomorphism, where $K$ depends only on $L$.

One proves the corollary by constructing a homeomorphism $(\lambda / 8) S \rightarrow J$ or $(\lambda / 2) I^{1} \rightarrow J$ with arc length as the parameter, and then using Theorems A and B.

4. Our proof is based on Carleson's method. This method was used in [3] to construct a quasiconformal extension to the upper half-space of $R^{4}$ of a quasiconformal homeomorphism of $R^{3}$. One can give an abstract definition of Carleson's method as follows. A similarity between metric spaces is a homeomorphism under which distances are multiplied by a constant. Two maps $f_{1}$ and $f_{2}$ are similar if there are similarities $g$ and $h$ such that $f_{2}=g f_{1} h$. Let $g: X \rightarrow Y$ be a map between metric spaces. If we make use of a sequence $g_{n}: X \rightarrow Y, n \geqq 0$, of maps with $\lim _{n \rightarrow \infty} g_{n}=g$ such that there are covers $A_{n i}, i \in I_{n}$, of $X$ with the property that the number of similarity classes of the maps $g_{n} \mid A_{n i}, n \geqq 0$ and $i \in I_{n}$, is finite, we say that Carleson's method is used.

The plan of our proof can be seen from Fig. 1. We consider a sequence $S_{i} \subset I^{2}$, $i \geqq 1$, of square-form circles converging to $S$ (in Fig. $1, S_{i}=\left(1-2^{-i}\right) S$ ). Let $R_{i}$ be the ring with boundary $S_{i} \cup S_{i+1}$. We divide each $R_{i}$ into quadrilaterals and obtain the left side of Fig. 1. Let $f: S \rightarrow R^{2}$ be the given $L$-embedding. We use Carleson's method, made possible by the compactness of the set of $L$-embeddings $g: I \rightarrow R^{2}$ with $\|g(0)\| \leqq 1$, to construct a sequence $f_{i}: S_{i} \rightarrow R^{2}$ of piecewise linear embeddings such that $f_{i}\left(x_{i}\right) \rightarrow f(x)$ whenever $x_{i} \rightarrow x, x_{i} \in S_{i}$. In addition, we assume that $f_{j}\left(S_{j}\right)$ is inside $f_{i}\left(S_{i}\right)$ if $i>j$ and all are inside $f(S)$ (cf. the right side of Fig. 1). Finally, we assume (this is the crux of the matter) that the number of similarity classes of $f_{i} \mid s, s$ a side of a quadrilateral in Fig. 1, is finite. Now we have an embedding $f \cup f_{1} \cup f_{2} \cup \ldots: S \cup S_{1} \cup S_{2} \cup \ldots \rightarrow R^{2}$. Next, we extend this embedding to all sides of the quadrilaterals in Fig. 1, i.e., on the "crossbars" between $S_{i}$ and 


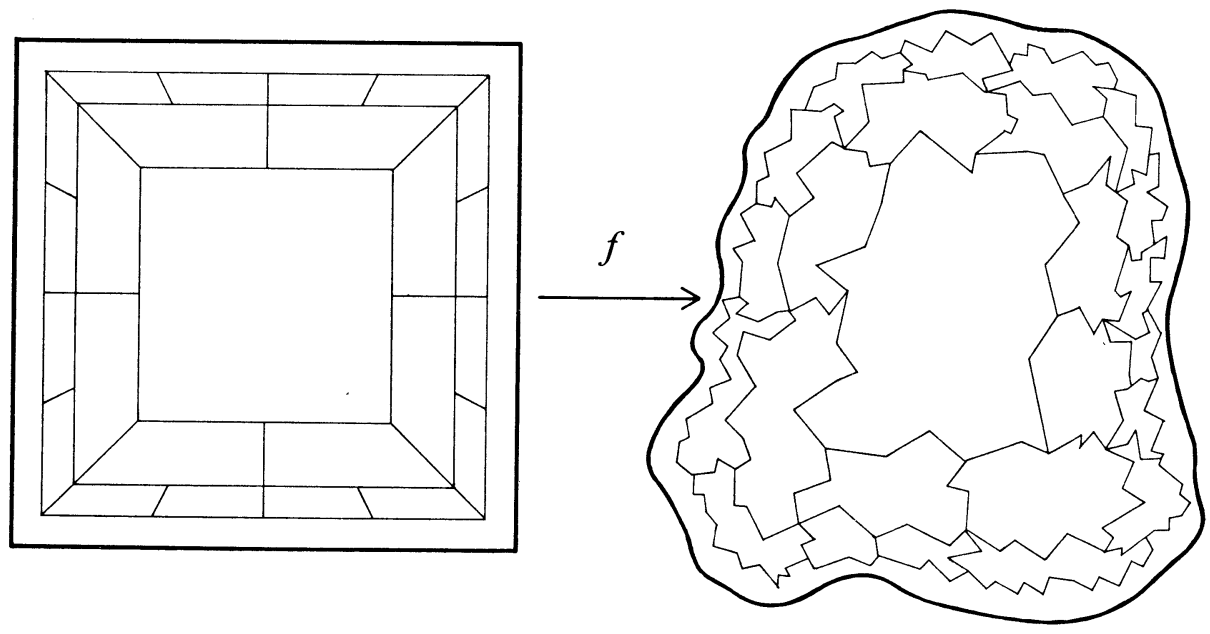

Figure 1

$S_{i+1}$, to obtain an embedding $f^{\prime}$ into $R^{2}$. We take care that the number of similarity classes of $f^{\prime}(J), J$ the boundary of a quadrilateral in Fig. 1, is finite. Since the PL Schönflies theorem is true in the plane, we can extend $f^{\prime}$ to an embedding $f^{\prime \prime}$ of $I^{2}$ in such a way that we again have a finiteness condition for the maps $f^{\prime \prime} \mid Q, Q$ a quadrilateral in Fig. 1. It turns out that $f^{\prime \prime}$ is a Lipschitz embedding extending $f$. Finally, we extend $f$ also on the outside of $I^{2}$, to obtain Theorem A.

5. In my opinion, Carleson's method is important. There probably are results that cannot be had otherwise. A drawback of the method is that in actual application it turns out to be quite laborious, and our paper is no exception to this. Another limitation of the method is, at least for the present, that it makes use of PL approximations that are known to exist only for dimensions $n \leqq 3$.

In addition to Carleson's paper [3] already mentioned, Carleson's method has been used in [14] and [11] to obtain some results in Lipschitz topology, such as the Lipschitz annulus conjecture and the Lipschitz hauptvermutung for $n \leqq 3$. It follows also from Theorem 2.4 of Väisälä [14] that if a Lipschitz embedding of $S$ into $R^{2}$ can be extended to a Lipschitz embedding of $I^{2}$, then there is also an extension which is PL in int $I^{2}$ and a Lipschitz embedding.

6. Theorem A was one of the open problems of Luukkainen-Väisälä [6, Section 9]. In the final chapter we also give a negative answer to a problem of [6, Section 9], by showing that there is a LIP arc $J_{0}$ in $R^{3}$ which is locally TOP flat but not locally LIP flat. We also propose a new conjecture relating to this problem.

In Section 17 we discuss the quasiconformal case. We show that the arc $J_{0}$ is not even locally quasiconformally flat and formulate some conjectures concerning quasiconformal flatness. 
7. We recall the notion of flatness which is used in the final chapter. Let $A \subset R^{n}$ and let $f: A \rightarrow R^{n}$ be an embedding. We say that $f$ is topologically flat if $f$ can be extended to an embedding of a neighbourhood of $A$ in $R^{n}$ into $R^{n}$. (If $A \subset R^{k}$ with $k<n$, we regard $A$ also as a subset of $R^{n}$.) If $x \in A, f$ is locally topologically flat at $x$ provided $x$ has a neighbourhood $V$ in $A$ such that $f \mid V$ is topologically flat. If $f$ is a Lipschitz embedding, the definitions of Lipschitz flatness and local Lipschitz flatness are similar. Finally, if $J \subset R^{n}$ is a (Lipschitz) arc, the meaning of " $J$ is (Lipschitz) flat" and " $J$ is locally (Lipschitz) flat" is clear.

8. I am indebted to J. Väisälä and J. Luukkainen for close reading of the manuscript, which resulted in many corrections and improvements. It was J. Luukkainen who insisted that every inaccuracy had to be removed. For instance, he made me aware of the need for condition (M) in Lemma 3A.

Added after the completion of this paper. Some time ago I found that one can prove a variant of Theorem A by aid of Riemann's mapping theorem and the Beurling-Ahlfors extension of a quasisymmetric map. This proof is much shorter. See "Extension of quasisymmetric and Lipschitz embeddings of the real line into the plane", to appear in these Annals.

\section{Proofs of Theorems A and B}

9. Lemma 1 is the variant of Moise's lemma needed (cf. [3, Lemma p. 44] and [14, Lemma 2.2]).

Let $J=[-1,2], J^{\prime}=[-2,3]$ and let $I_{i}=[i-1,1]$ for $i \in Z$. In the following, families of maps are topologized by means of the topology of uniform convergence. In Lemma 1 (a) and (c) $\mathscr{F}$ is a compact family of embeddings $J \rightarrow R^{2}$ and in (b) $\mathscr{F}^{\prime}$ is a compact family of embeddings $J^{\prime} \rightarrow R^{2}$. It is assumed that $\varepsilon>0$ is given such that it satisfies conditions (i) -(iii) below. $\left(U_{\varepsilon}(z)\right.$ is the open ball with radius $\varepsilon$ and center $z$.)

(i) $U_{\varepsilon}(f(x)) \cap U_{\varepsilon}(f(y))=\emptyset$ if $x \leqq 0, y \geqq 1$ for $f \in \mathscr{F} \cup \mathscr{F}^{\prime}$.

(ii) Let $\quad U_{f}=U_{f, \varepsilon}=\bigcup_{x \in I_{1}} U_{\varepsilon}(f(x))$. Then, if $f \in \mathscr{F} \cup \mathscr{F}^{\prime}, \quad U_{f} \backslash \operatorname{im} f$ has exactly two components $C_{f}^{1}$ and $C_{f}^{2}$ such that $f\left(I_{1}\right) \subset \mathrm{cl} C_{f}^{i}, i=1,2$. If $f \in \mathscr{F}, f^{\prime} \in \mathscr{F}^{\prime}$ and $f(x)=f^{\prime}(x-1)$ or $f(x)=f^{\prime}(x+1)$ for $x \in J$, then $C_{f}^{t}, t=1,2$, intersects only one of the components $C_{f}^{1}$, and $C_{f}^{2}$.

(iii) Let $f \in \mathscr{F} \cup \mathscr{F}^{\prime}$, and let $g: S \rightarrow R^{2}$ be a continuous map such that, when $C^{1}$. and $C_{f}^{2}$ are as in (ii),

$$
\begin{gathered}
g\left(\{1\} \times I^{1}\right) \subset U_{\varepsilon}(f(1)), g\left(\{-1\} \times I^{1}\right) \subset U_{\varepsilon}(f(0)), \\
g\left(I^{1} \times\{1\}\right) \subset C_{f}^{t^{\prime}}, g\left(I^{1} \times\{-1\}\right) \subset C_{f}^{t^{\prime \prime}}, \quad \text { where } t^{\prime}, t^{\prime \prime} \in\{1,2\} .
\end{gathered}
$$


Then $f(1 / 2) \notin g(S)$ and $g$ turns round $f(1 / 2) \quad t^{\prime \prime}-t^{\prime}$ times (i.e.,

$$
\int_{S}(1 /(g(z)-f(1 / 2))) d g(z)=\left(t^{\prime \prime}-t^{\prime}\right) 2 \pi i,
$$

where $S$ has the natural orientation).

Note that in (ii) there may be more than two components but not more than two of them come close to $f\left(I_{1}\right)$, and that (iii) fixes the notation $C_{f}^{1}$ and $C_{f}^{2}$ for the components ( $C_{f}^{1}$ is the "left" side and $C_{f}^{2}$ is the "right" side). It is easy to see that there is $\varepsilon_{0}>0$ such that (i)-(iii) is satisfied for $0<\varepsilon \leqq \varepsilon_{0}$. For instance, in (ii) we can reason as follows. Since embeddings of arcs in $R^{2}$ are flat, it follows easily that for every $f \in \mathscr{F} \cup \mathscr{F}^{\prime}$ there is $\varepsilon_{f}>0$ such that $U_{f, \varepsilon_{f}} \backslash \operatorname{im} f$ has two such components $C_{f}^{1}$ and $C_{f}^{2}$. But now $f$ has a neighbourhood $\mathscr{U}$ in $\mathscr{F}$ (or in $\mathscr{F}^{\prime}$ ) such that $U_{f^{\prime}, \varepsilon_{f} / 2} \backslash \operatorname{im} f^{\prime}, f^{\prime} \in \mathscr{U}$, has also two such components. The result follows by compactness. Similarly, (iii) follows by flatness and by compactness.

In Lemma 1 we need only conditions (i) and (ii). Condition (iii) is needed later for Theorem $\mathbf{B}$ in Lemmas $2 \mathrm{~B}$ and $3 \mathrm{~B}$.

Lemma 1. (a) We can express $\mathscr{F}$ as a union $\mathscr{F}=\mathscr{F}_{1} \cup \ldots \cup \mathscr{F}_{k}$, where each $\mathscr{F}_{i}$ is compact, and find $\mathrm{PL}$ embeddings $g_{1}^{t}, \ldots, g_{k}^{t}: I_{1} \rightarrow R^{2}, t=1,2$, such that if $f \in \mathscr{F}_{i}, t=1,2$,

$$
\begin{gathered}
g_{i}^{t}\left(I_{1}\right) \subset C_{f}^{t}, \\
\left\|g_{i}^{t}(x)-f(x)\right\|<\varepsilon \quad \text { for } \quad x \in I_{1}, \\
g_{i}^{t}\left(I_{1}\right) \cap f(J)=\emptyset .
\end{gathered}
$$

(b) There is $\varepsilon^{\prime}>0, \varepsilon^{\prime}<\varepsilon$, depending only on $\varepsilon$ and $\mathscr{F}^{\prime}$, with the following properties. Assume that we have expressed $\mathscr{F}^{\prime}$ as a union $\mathscr{F}^{\prime}=\mathscr{F}_{1}^{\prime} \cup \ldots \cup \mathscr{F}_{k^{\prime}}$, of compact sets and that there are PL embeddings $h_{1}^{t}, \ldots, h_{k^{\prime}}^{t}: I_{0} \cup I_{2} \rightarrow R^{2}, t=1,2$, such that for every $f \in \mathscr{F}_{i}^{\prime}, t=1,2$,

$$
\begin{gathered}
h_{i}^{t}(0), h_{i}^{t}(1) \in C_{f}^{t}, \\
\left\|h_{i}^{t}(x)-f(x)\right\|<\varepsilon^{\prime} \quad \text { for } \quad x \in I_{0} \cup I_{2}, \quad \text { and } \\
h_{i}^{t}\left(I_{0} \cup I_{2}\right) \cap f\left(J^{\prime}\right)=\emptyset .
\end{gathered}
$$

Then we can express every $\mathscr{F}_{i}^{\prime}$ as a union $\mathscr{F}_{i}^{\prime}=\mathscr{F}_{i 1}^{\prime} \cup \ldots \cup \mathscr{F}_{i n_{i}}^{\prime}$ of compact sets and find PL embeddings $h_{i 1}^{t}, \ldots, h_{i n_{i}}^{t}: J \rightarrow R^{2}, t=1,2$, extending $h_{i}^{t}$ such that for every $f \in \mathscr{F}_{i j}^{\prime}$, $t=1,2$,

$$
\begin{gathered}
\left\|h_{i j}^{t}(x)-f(x)\right\|<\varepsilon \quad \text { for } \quad x \in J, \quad \text { and } \\
h_{i j}^{t}(J) \cap f\left(J^{\prime}\right)=\emptyset .
\end{gathered}
$$

(c) We can express $\mathscr{F}$ as a union $\mathscr{F}=\mathscr{F}_{1}^{\prime \prime} \cup \ldots \cup \mathscr{F}_{k \prime \prime}^{\prime \prime}$, where each $\mathscr{F}_{i}^{\prime \prime}$ is compact, and find PL embeddings $g_{1}, \ldots, g_{k^{\prime \prime}}: A=I_{2} \times\{ \pm 1\} \cup\{2\} \times I^{1} \rightarrow R^{2}$ such that if $f \in \mathscr{F}_{i}^{\prime \prime}$,

$$
\begin{gathered}
g_{i}(1,1) \in C_{f}^{1} \text { and } g_{i}(1,-1) \in C_{f}^{2}, \\
\left\|g_{i}(x, y)-f(x)\right\|<\varepsilon \text { for }(x, y) \in A, \\
g_{i}(A) \cap f(J)=\emptyset .
\end{gathered}
$$


Note. For Theorem A we need only (a) and (b); for Theorem B we need also (c) which is an analogue of (a) used in the proof of Theorem B to turn around the endpoints of the image of the interval $I^{1}$. There is also an analogue for (b) in this case but we can arrange the proof of Theorem B in such a way that it is not needed.

Proof of (a). Let $f \in \mathscr{F}$. Since embeddings of intervals into $R^{2}$ are flat, there is a homeomorphism $F: R^{2} \rightarrow R^{2}$ extending $f$. Since we can approximate homeomorphisms of 2-manifolds by PL homeomorphisms (apply Moise [7, Theorem 3 of Section 6] to $F \mid R^{2} \backslash J$ with a suitable $\varepsilon: R^{2} \backslash J \rightarrow R_{+}$), we can assume that $F$ is PL outside $J$. Now there is $u \neq 0$ such that the maps $g_{f}^{t}: I_{1} \rightarrow R^{2}, g_{f}^{t}(x)=$ $F\left(x,(-1)^{t} u\right), t=1,2$, satisfy $(\alpha)$ with $g_{i}^{t}=g_{f}^{t}$. Clearly, there is also a neighbourhood $\mathscr{U}$ of $f$ in $\mathscr{F}$ such that $(\alpha)$ is satisfied for every $f^{\prime} \in \operatorname{cl} \mathscr{U}$ with $g_{i}^{t} \mapsto g_{f}^{t}$ and $f \mapsto f^{\prime}$. Let $A=\left\{\left(\mathscr{U}, g^{1}, g^{2}\right): \mathscr{U} \subset \mathscr{F}\right.$ open, $g^{1}$ and $g^{2}$ PL embeddings $I_{1} \rightarrow R^{2}$ such that $(\alpha)$ is satisfied for every $f \in \mathrm{cl} \mathscr{U}$ with $g_{i}^{t} \rightarrow g^{t}$ \}. We have shown that if $f \in \mathscr{F}$, there is $\left(\mathscr{U}, g^{1}, g^{2}\right) \in A$ such that $f \in \mathscr{U}$. Therefore (a) is true by compactness.

Proof of (b). By compactness of $\mathscr{F}^{\prime}$ we can find $\bar{\varepsilon}>0, \bar{\varepsilon} \leqq \varepsilon$, such that if $x, y \in J^{\prime}$ and $f \in \mathscr{F}^{\prime}$, then $\|f(x)-f(y)\| \leqq 2 \bar{\varepsilon}$ implies $f([x, y]) \subset U_{\varepsilon / 2}(f(x))$. Repeating this argument, we can find $\varepsilon^{\prime}>0,4 \varepsilon^{\prime}<\bar{\varepsilon}$, such that if $x, y \in J^{\prime}$ and $f \in \mathscr{F}^{\prime}$, then $\|f(x)-f(y)\| \leqq 2 \varepsilon^{\prime}$ implies $f([x, y]) \subset U_{\tilde{\varepsilon} / 4}(f(x))$. Clearly, $\varepsilon^{\prime}$ depends only on $\varepsilon$ and $\mathscr{F}^{\prime}$, and we show that (b) is true with this $\varepsilon^{\prime}$.

First we note that if $f \in \mathscr{F}^{\prime}, C=C_{f}^{t}, t=1,2, x \in I_{1}$ and $y, z \in U_{\varepsilon^{\prime}}(f(x)) \cap C$, $y \neq z$, we can join $y$ and $z$ by a PL arc in $U_{\bar{\varepsilon}}(f(x)) \cap C$. To see this, let $y z$ be the line segment joining $y$ and $z$. Then $y z \subset U_{\varepsilon^{\prime}}(f(x))$, and if $y z \cap f\left(J^{\prime}\right)=\emptyset$, there is nothing more to be proved. Otherwise, let $y^{\prime}, z^{\prime} \in y z$ be the points such that $y y^{\prime} \cap$ $f\left(J^{\prime}\right)=\left\{y^{\prime}\right\}$ and $z z^{\prime} \cap f\left(J^{\prime}\right)=\left\{z^{\prime}\right\}$. Then $\left\|y^{\prime}-z^{\prime}\right\|<2 \varepsilon^{\prime}$, implying that the diameter of the subarc of $f\left(J^{\prime}\right)$ with endpoints $y^{\prime}$ and $z^{\prime}$ is less than $\bar{\varepsilon} / 2$. Now, as in the proof of (a), let $F$ be an extension of $f$ to a homeomorphism of $R^{2}$ onto $R^{2}$ such that $F$ is PL outside $J^{\prime}$. Since $y$ and $z$ are on the same side of $f\left(J^{\prime}\right)$ (i.e. in $C$ ), it is easy to see, using the map $F$, that there is a PL arc $J_{y z} \subset C \cap U_{\bar{\varepsilon}}(f(x))$ joining $y$ and $z$. The arc $J_{y z}$ consists of a subsegment of $y y^{\prime}$, of a subsegment of $z z^{\prime}$ and of a PL arc close to a subarc of $f\left(J^{\prime}\right)$.

We fix now $i \leqq k^{\prime}, t=1,2$, and choose $f \in \mathscr{F}_{i}^{\prime}$. Then $h_{i}^{t}(0) \in C=C_{f}^{t}$. Let $F: R^{2} \rightarrow R^{2}$ be an extension of $f$ to a homeomorphism of $R^{2}$ as above. Then, for some $u \neq 0$, the map $g_{f}^{t}: I_{1} \rightarrow R^{2}, g_{f}^{t}(x)=F(x, u)$, defines a PL embedding $I_{1} \rightarrow R^{2} \backslash f\left(J^{\prime}\right)$ such that $\left\|g_{f}^{t}(x)-f(x)\right\|<\varepsilon^{\prime}, g_{f}^{t}\left(I_{1}\right) \cap h_{i}^{t}\left(I_{0} \cup I_{2}\right)=\emptyset$, and that $g_{f}^{t}\left(I_{1}\right) \subset C$. The definition of $\bar{\varepsilon}$ implies also that we can assume that if $\left\|f(x)-g_{f}^{t}(y)\right\| \leqq 2 \bar{\varepsilon}, x, y \in I_{1}$, then $g_{f}^{t}([x, y]) \subset U_{\varepsilon / 2}(f(x))$. (To see this, note that actually there is $\varepsilon_{1}>2 \bar{\varepsilon}$ such that $g \in \mathscr{F}^{\prime}$ and $\|g(x)-g(y)\| \leqq \varepsilon_{1}$ imply $g([x, y]) \subset U_{\varepsilon / 2}(g(x))$.)

We consider the points $g_{f}^{t}(0)$ and $h_{i}^{t}(0)$. They are both in $U_{\varepsilon^{\prime}}(f(0)) \cap C$. Therefore there is a PL arc $J_{0}$ in $U_{\bar{\varepsilon}}(f(0)) \cap C$ joining $g_{f}^{t}(0)$ and $h_{i}^{t}(0)$. Then $J_{0}$ has a subarc $J_{0}^{\prime}$ such that $J_{0}^{\prime} \cap g_{f}^{t}\left(I_{1}\right)=\left\{g_{f}^{t}(x)\right\}$ and $J_{0}^{\prime} \cap h_{i}^{t}\left(I_{0}\right)=\left\{h_{i}^{t}(y)\right\}, x \in I_{1}$ and $y \in I_{0}$. Therefore there is a PL arc $J_{0}^{\prime \prime}$ consisting of a subarc $J_{1}$ of $J_{0}^{\prime}$, of an arc $J_{2}$ close to 
$g_{f}^{t}([0, x])$ and of an arc $J_{3}$ close to $h_{i}^{t}([y, 0])$, such that $J_{0}^{\prime \prime} \cap g_{f}^{t}\left(I_{1}\right)=\left\{g_{f}^{t}(0)\right\}$ and $J_{0}^{\prime \prime} \cap h_{i}^{t}\left(I_{0}\right)=\left\{h_{i}^{t}(0)\right\}$. We claim that $J_{0}^{\prime \prime} \subset U_{\varepsilon}(f(0)) \cap C$. It is clear that $J_{1} \subset U_{\varepsilon}(f(0)) \cap C$. Since $\left.\| g_{f}^{t}(x)-f(0)\right)<\bar{\varepsilon}, g_{f}^{t}([0, x]) \subset U_{\varepsilon / 2}(f(0))$. Therefore if $J_{2}$ is sufficiently close to $g_{f}^{t}([0, x])$, also $J_{2} \subset U_{\varepsilon}(f(0)) \cap C$, which we now assume. Since $\left\|h_{i}^{t}(y)-f(0)\right\|<\bar{\varepsilon}$ and $\left\|h_{i}^{t}(y)-f(y)\right\|<\varepsilon^{\prime}<\bar{\varepsilon}$, we have $\|f(0)-f(y)\|<2 \bar{\varepsilon}$, and therefore $f([y, 0]) \subset$ $U_{\varepsilon / 2}(f(0))$, implying that $h_{i}^{t}([y, 0]) \subset U_{\varepsilon}(f(0))$. Consequently, we can assume that $J_{3}$ is so close to $h_{i}^{t}([y, 0])$ that $J_{3} \subset U_{\varepsilon}(f(0)) \cap C$. Since $J_{0}^{\prime \prime}=J_{1} \cup J_{2} \cup J_{3}$, we have $J_{0}^{\prime \prime} \subset U_{\varepsilon}(f(0)) \cap C$.

In the same manner, since $h_{i}^{t}(1) \in C$, we can find a PL arc $J_{1}^{\prime \prime}$ in $U_{\varepsilon}(f(1)) \cap C$ such that $J_{1}^{\prime \prime} \cap g_{f}^{t}\left(I_{1}\right)=\left\{g_{f}^{t}(1)\right\}$ and $J_{1}^{\prime \prime} \cap h_{i}^{t}\left(I_{2}\right)=\left\{h_{i}^{t}(1)\right\}$. By $(\mathrm{i})$, since $\left\|h_{i}^{t}(x)-f(x)\right\|<\varepsilon^{\prime} \leqq \varepsilon$, $J_{0}^{\prime \prime} \cup J_{1}^{\prime \prime} \cup h_{i}^{t}\left(I_{0} \cup I_{2}\right) \cup g_{f}^{t}\left(I_{1}\right)$ is a PL arc, and it is easy to see that there is a parametrization of it as a map $h_{f}^{t}: J \rightarrow R^{2}$ in such a way that $h_{f}^{t}$ extends $h_{i}^{t}$ and satisfies $(\beta)$ if we set in it $h_{i j}^{t}=h_{f}^{t}$. It is clear that $(\beta)$ is also satisfied with the substitutions $f_{\mapsto} \mapsto f^{\prime}$ and $h_{i j}^{t} \mapsto h_{f}^{t}$ when $f^{\prime} \in \operatorname{cl} \mathscr{U}$, where $\mathscr{U}$ is some neighbourhood of $f$ in $\mathscr{F}_{i}^{\prime}$. This implies (b) by compactness; see the proof of (a).

Proof of (c) is similar to the proof of (a) and is omitted.

10. In this section we construct, given an $L$-embedding $f: S \rightarrow R^{2}$, PL embeddings $f_{n}: S \rightarrow R^{2}, n \geqq 1$, such that the sequence $f_{n}(S), n \geqq 1$, has a subsequence which consists of concentric PL circles converging towards $f(S)$ in the bounded component of $R^{2} \backslash f(S)$ (cf. Fig. 1).

We denote by $K_{h}, h \geqq 0$, the triangulation of $S$ such that an interval $s \subset S$ is a 1-simplex of $K_{h}$ if and only if $s$ has length $2^{-h}$ and the endpoints of $s$ are of the form $\left(i / 2^{h}, j / 2^{h}\right), i, j \in Z$.

Lemma 2A. Let $f: S \rightarrow R^{2}$ be an L-embedding and let $C$ be the bounded component of $R^{2} \backslash f(S)$. Then there are PL embeddings $f_{n}: S \rightarrow C, n \geqq 1$, such that (A1) $\left\|f_{n}(x)-f(x)\right\|<2^{-n} / 4 L$ for $x \in S$, and

(A2) $\left\|f_{n}(x)-f(y)\right\| \geqq \delta 2^{-n}$ for $x, y \in S$,

where $\delta>0$ depends only on $L$. In addition we have:

(A3) The similarity classes of the maps $f_{n} \mid s \cup s^{\prime}$, when $s$ and $s^{\prime}$ are two adjacent 1-simplexes of $K_{n}, n \geqq 1$, are in a finite set depending only on $L$.

(A4) The similarity classes of the triangles (possibly degenerated) with vertices $f_{n}(u)$, $f_{n}(v)$ and $f_{n+1}(u)$, when $u$ and $v$ are the endpoints of a 1-simplex of $K_{n}, n \geqq 1$, are in a finite set depending only on $L$.

Finally,

$$
1 / 2 \leqq \frac{\|f(u)-f(v)\|}{\left\|f_{n}(u)-f_{n}(v)\right\|} \leqq 2
$$

whenever $u$ and $v$ are the endpoints of a 1-simplex of $K_{n}, n \geqq 1$. 
For Theorem B, we need a slightly different version of Lemma $2 \mathrm{~A}$. If $f^{\prime}: I^{1} \rightarrow R^{2}$ is the given embedding, we encircle $f^{\prime}\left(I^{1}\right)$ by concentric PL circles. Let

$$
S_{n}^{\prime}=\partial\left(I^{1} \times\left[-2^{-n}, 2^{-n}\right]\right)
$$

and let $\bar{K}_{n}$ be the triangulation of $S_{n}^{\prime}$ that has $4\left(2^{n}+1\right) 1$-simplexes of length $2^{-n}$.

Lemma 2B. Let $f^{\prime}: I^{1} \rightarrow R^{2}$ be an L-embedding. Then there are $\delta^{\prime}>0$, depending only on $L$, and PL embeddings $f_{n}^{\prime}: S_{n}^{\prime} \rightarrow R^{2} \backslash f^{\prime}\left(I^{1}\right), n \geqq 1$, such that, if $x \in I^{1}$, each $f_{n}^{\prime}$ turns round $f^{\prime}(x)$ once in the positive direction, and that

$$
\begin{gathered}
\left\|f_{n}^{\prime}(x, y)-f^{\prime}(x)\right\|<2^{-n} / 4 L \quad \text { for } \quad(x, y) \in S_{n}^{\prime}, \quad \text { and } \\
\left\|f_{n}^{\prime}(x)-f^{\prime}(y)\right\| \geqq \delta^{\prime} 2^{-n}, \quad x \in S_{n}^{\prime}, y \in I^{1} .
\end{gathered}
$$

Also (A3) and (A4) remain true if we substitute in them $f_{n} \mapsto f_{n}^{\prime}$ and $K_{n} \mapsto \bar{K}_{n}$. These are referred to as (B3) and (B4). Finally,

$$
1 / 2 \leqq \frac{\|f(u)-f(v)\|}{\left\|f_{n}^{\prime}(u, t)-f_{n}^{\prime}(v, t)\right\|} \leqq 2
$$

whenever $(u, t)$ and $(v, t)$ are the endpoints of a 1-simplex of $\bar{K}_{n}, n \geqq 1$.

Proof. We prove Lemma $2 \mathrm{~A}$ and after it make some remarks concerning the proof of Lemma $2 \mathrm{~B}$, which is essentially similar.

In order to apply Lemma 1 we must take care of the corners of the square-form circle $S$; for this we make the following observation. Let $r:[-1,3] \rightarrow S$ be defined by $x \mapsto(x,-1)$ if $|x| \leqq 1, x \mapsto(1, x-2)$ if $|x-2| \leqq 1$. Then $r$ is a $\sqrt{2}$-embedding, and, consequently, if $g: S \rightarrow R^{2}$ is an $L_{1}$-embedding, $g r$ is a $\sqrt{2} L_{1}$-embedding. Let the family $\mathscr{F}$ in Lemma 1 (a) be the family of $\sqrt{2} L$-embeddings $g: J=[-1,2] \rightarrow R^{2}$ such that $\|g(0)\| \leqq 1$, and let the family $\mathscr{F}^{\prime}$ in Lemma 1 (b) be the family of $\sqrt{2} L$ embeddings $g^{\prime}: J^{\prime}=[-2,3] \rightarrow R^{2}$ such that $\left\|g^{\prime}(0)\right\| \leqq 1$. They are compact families. There is a number $\varepsilon>0, \varepsilon \leqq 1 / 4 L$, that satisfies conditions (i)-(iii) of Section 9 with respect to this $\mathscr{F}$ and $\mathscr{F}^{\prime}$. Let $\varepsilon^{\prime}>0, \varepsilon^{\prime} \leqq \varepsilon / 2 L^{2}$, be the number obtained in Lemma 1 (b) with this $\varepsilon$ and $\mathscr{F}^{\prime}$. Then $\varepsilon$ and $\varepsilon^{\prime}$ are functions of $L$ only. We require $\varepsilon \leqq 1 / 4 L$ primarily for the following reason. If $g: R \rightarrow R^{2}$ is a $\sqrt{2} L$-embedding and $g^{\prime}: R \rightarrow R^{2}$ is an $\varepsilon$-approximation of $g$ (not necessarily an embedding), then for any $x, y \in R,|x-y| \geqq 1$, we have $g^{\prime}(x) \neq g^{\prime}(y)$.

Next we define $\sqrt{2} L$-embeddings $f_{s}: J \rightarrow R^{2}$ and $f_{s}^{\prime}: J^{\prime} \rightarrow R^{2}$ for every 1-simplex $s$ of $K_{n}, n \geqq 1$. For every such $s$, let $s_{1}, s_{2}, s_{3}=s, s_{4}$ and $s_{5}$ be the five 1-simplexes of $K_{n}$ such that $s_{i+1}$ is adjacent to $s_{i}$ and that the sequence $\left(s_{1}, s_{2}, s_{3}, s_{4}, s_{5}\right)$ is coherently oriented with respect to $S$. Let $p_{s}: J^{\prime} \rightarrow S$ be defined by the requirements that $p_{s} \mid[i-1, i]$ is affine and that $p_{s}([i-1, i])=s_{i+2}$. We choose a point 
$a_{s} \in R^{2}$ with coordinates $\left(i / 2^{n}, j / 2^{n}\right), i, j \in Z$, such that $\left\|f\left(p_{s}(0)\right)-a_{s}\right\| \leqq 1 / 2^{n}$. Let $q_{s}: R^{2} \rightarrow R^{2}$ be the map $x \mapsto 2^{n}\left(x-a_{s}\right)$. Then we define $f_{s}: J \rightarrow R^{2}$ and $f_{s}^{\prime}: J^{\prime} \rightarrow R^{2}$ by

$$
\begin{aligned}
& f_{s}^{\prime}=q_{s} \circ f \circ p_{s}, \\
& f_{s}=f_{s}^{\prime} \mid J .
\end{aligned}
$$

Then each $f_{s}$ and $f_{s}^{\prime}$ is a $\sqrt{2} L$-embedding. Also $\left\|f_{s}(0)\right\|=\left\|f_{s}^{\prime}(0)\right\| \leqq 1$. It follows that $\left\{f_{s}: s \in K_{n}, n \geqq 1\right\} \subset \mathscr{F}$ and $\left\{f_{s}^{\prime}: s \in K_{n}, n \geqq 1\right\} \subset \mathscr{F}^{\prime}$. Since $\varepsilon \leqq 1 / 4 L, q_{s}^{-1}\left(U_{f_{s}}\right) \cap$ $f\left(S \backslash\left(s_{2} \cup S_{3} \cup S_{4}\right)\right)=\emptyset$ for $s \in K_{n}, n \geqq 1$. Therefore $C_{f_{s}}^{t}=C_{f_{s}^{\prime}}^{t}, t=1,2$, and $q_{s}^{-1}\left(C_{f_{s}}^{1}\right) \subset C$ or $q_{s}^{-1}\left(C_{f_{s}}^{2}\right) \subset C$ for all $s \in K_{n}, n \geqq 1$. We can assume $q_{s}^{-1}\left(C_{f_{s}}^{1}\right)=q^{-1}\left(C_{f_{s}^{\prime}}^{1}\right) \subset C$.

We define $f_{n}$ in two stages. We divide the set of 1-simplexes of $K_{n}$ into two disjoint sets $K_{n}^{\prime}$ and $K_{n}^{\prime \prime}$ in such a way that if $s$ and $s^{\prime}$ are both in $K_{n}^{\prime}$ or in $K_{n}^{\prime \prime}$, then $s \cap s^{\prime}=\emptyset$ if $s \neq s^{\prime}$. We can, for instance, set that the simplex with endpoints $(1,1)$ and $\left(1-2^{-n}, 1\right)$ is in $K_{n}^{\prime}$. This defines $K_{n}^{\prime}$ and $K_{n}^{\prime \prime}$ uniquely. We define $f_{n}$ first in $\cup K_{n}^{\prime}$ and then in $\cup K_{n}^{\prime \prime}$.

First we express $\mathscr{F}$ as a union $\mathscr{F}=\mathscr{F}_{1} \cup \ldots \cup \mathscr{F}_{k}$ and find the maps $g_{1}^{t}, \ldots, g_{k}^{t}$, $t=1,2$, as in Lemma 1 (a), where we have substituted $\varepsilon \mapsto \varepsilon^{\prime}$. Now we can define

$$
f_{n}\left|s=q_{s}^{-1} \circ g_{i}^{1} \circ p_{s}^{-1}\right| s \quad \text { when } \quad s \in K_{n}^{\prime},
$$

where $i=i(s)$ is chosen in such a way that $f_{s} \in \mathscr{F}_{i}$. Since $q_{s}^{-1}\left(C_{f_{s}}^{1}\right) \subset C$, we have $f_{n}(s) \subset C$ for all $s \in K_{n}^{\prime}$.

To extend $f_{n}$ to $\cup K_{n}^{\prime \prime}$ we must find the families $\mathscr{F}_{1}^{\prime}, \ldots, \mathscr{F}_{k^{\prime}}^{\prime}$ and maps $h_{1}^{t}, \ldots, h_{k^{\prime}}^{t}$ of Lemma 1 (b). If $i, j \leqq k$ and $a, b \in Z^{2}$, we let $\mathscr{F}_{i j a b}$ be the subset of $\mathscr{F}^{\prime}$ for which $g \in \mathscr{F}_{i j a b}$ if and only if

$$
\begin{array}{ll}
\text { the map } g_{a}^{+}: J \rightarrow R^{2}, & x \mapsto g(x+1)-a, \text { is in } \mathscr{F}_{i}, \quad \text { and } \\
\text { the map } g_{b}^{-}: J \rightarrow R^{2}, & x \mapsto g(x-1)-b, \text { is in } \mathscr{F}_{j} .
\end{array}
$$

Note that only a finite number of the families $\mathscr{F}_{i j a b} \neq \emptyset$. The non-empty families $\mathscr{F}_{i j a b}$ we label as $\mathscr{F}_{1}^{\prime}, \ldots, \mathscr{F}_{k^{\prime}}$, where to each quadruple $(i, j, a, b) \neq\left(i^{\prime}, j^{\prime}, a^{\prime}, b^{\prime}\right)$ correspond distinct indices even if $\mathscr{F}_{i j a b}=\mathscr{F}_{i^{\prime} j^{\prime} a^{\prime} b^{\prime}}$. It is easy to see that $\mathscr{F}^{\prime}=\mathscr{F}_{1}^{\prime} \cup \ldots$ $\cup \mathscr{F}_{k^{\prime}}^{\prime}$ and that each $\mathscr{F}_{i}^{\prime}$ is compact. The maps $h_{r}^{t}: I_{0} \cup I_{2} \rightarrow R^{2}, r \leqq k^{\prime}$, we define by setting, if $\mathscr{F}_{r}^{\prime}=\mathscr{F}_{i j a b}$,

$$
\begin{gathered}
h_{r}^{t}(x)=g_{i}^{t}(x-1)+a, \quad x \in I_{2}, \quad \text { and } \\
h_{r}^{t}(x)=g_{j}^{t}(x+1)+b, \quad x \in I_{0} .
\end{gathered}
$$

We show that $h_{r}^{t}, r \leqq k^{\prime}, t \in\{1,2\}$, are PL embeddings $I_{0} \cup I_{2} \rightarrow R^{2}$ that satisfy the conditions $\left(\beta_{0}\right)$ of Lemma 1 (b). Clearly, every $h_{r}^{t}$ is PL and it is an embedding since $h_{r}^{t} \mid I_{i}, i=0,2$, is an embedding, since every $g \in \mathscr{F}_{r}^{\prime}$ is a $\sqrt{2} L$-embedding, and since $\varepsilon^{\prime} \leqq \varepsilon \leqq 1 / 4 L$. The last two conditions of $\left(\beta_{0}\right)$ follow from the fact the embeddings $g_{i}^{t}$ satisfy similar conditions (cf. $(\alpha)$ of Lemma $1(\mathrm{a})$ ) and that maps $f^{\prime} \in \mathscr{F}^{\prime}$ are 
$\sqrt{2} L$-embeddings. We show that $h_{r}^{t}(0), h_{r}^{t}(1) \in C_{g}^{t}$ for all $g \in \mathscr{F}_{r}^{\prime}$. We consider only $h_{r}^{t}(0)$ and fix $g \in \mathscr{F}_{r}^{\prime}$. We show that there is an arc $J_{0}$ in $U_{\varepsilon}(g(0))$ joining $g(0)$ and $h_{r}^{t}(0)$ with $J_{0} \cap g\left(J^{\prime}\right)=\{g(0)\}$. Let $s$ be the line segment joining $h_{r}^{t}(0)$ and $g(0)$. Let $s^{\prime} \subset s$ be the subsegment with one endpoint $h_{r}^{t}(0)$ and such that $s^{\prime} \cap g\left(J^{\prime}\right)=$ $\{g(x)\}, x \in J^{\prime}$. Then $|x-0|<\sqrt{2} L \varepsilon^{\prime}$ since $\|g(x)-g(0)\|<\varepsilon^{\prime}$. This implies that $g([x, 0]) \subset U_{2 L^{2} \varepsilon^{\prime}}(g(0)) \subset U_{\varepsilon}(g(0))$ since $2 L^{2} \varepsilon^{\prime} \leqq \varepsilon$. Therefore the required arc $J_{0}$ exists. Since $J_{0} \backslash\{g(0)\} \subset U_{\varepsilon}(g(0)) \backslash g\left(J^{\prime}\right)$, we have $J_{0} \backslash\{g(0)\} \subset C_{g}^{t^{\prime}}$, where $t^{\prime}=1$ or $t^{\prime}=2$. We also must have $J_{0} \backslash\{g(0)\} \subset C_{g^{\prime}}^{t^{\prime}}+b$, where $g^{\prime}=g_{b}^{-}$if $\mathscr{F}_{r}^{\prime}=\mathscr{F}_{i j a b}$. Since $h_{r}^{t}(0) \in C_{g^{\prime}}^{t}+b, t^{\prime}=t$. This proves that $h_{r}^{t}(0) \in C_{g}^{t}$. Therefore we can express each $\mathscr{F}_{r}^{\prime}$ as a union $\mathscr{F}_{r}^{\prime}=\mathscr{F}_{r 1}^{\prime} \cup \ldots \cup \mathscr{F}_{r n_{r}}^{\prime}$ and find PL embeddings $h_{r p}^{t}$ as in Lemma 1 (b). Note that the constructions in this and the preceding paragraph depend only on $L$.

Now we are ready to define $f_{n}$ in $\cup K_{n}^{\prime \prime}$. Let $s \in K_{n}^{\prime \prime}$ and let $s^{\prime}$ and $s^{\prime \prime} \in K_{n}^{\prime}$ be the 1-simplexes adjacent to $s, s^{\prime}$ coming after $s$. Then by (2), if $x \in J$,

$$
\begin{aligned}
f_{s^{\prime}}(x) & =q_{s^{\prime}} \circ f \circ p_{s^{\prime}}(x)=2^{n}\left(f\left(p_{s}(x+1)\right)-a_{s^{\prime}}\right) \\
& =q_{s} \circ f \circ p_{s}(x+1)-2^{n}\left(a_{s^{\prime}}-a_{s}\right) \\
& =\left(f_{s}^{\prime}\right)_{a}^{+}(x),
\end{aligned}
$$

where $a=2^{n}\left(a_{s^{\prime}}-a_{s}\right) \in Z^{2}$. In the same manner one has $f_{s^{\prime \prime}}=\left(f_{s}^{\prime}\right)_{b}^{-}$, where $b=2^{n}\left(a_{s^{\prime \prime}}-a_{s}\right)$. Let $i=i\left(s^{\prime}\right)$ and $j=i\left(s^{\prime \prime}\right)$ be the numbers used in (3) to define $f_{n} \mid s^{\prime}$ and $f_{n} \mid s^{\prime \prime}$. Then $f_{s}^{\prime} \in \mathscr{F}_{i j a b}=\mathscr{F}_{r}^{\prime}$ for some $r \leqq k^{\prime}$. Now, we have by (3), if $x \in I_{1}$,

$$
\begin{aligned}
& g_{i}^{1}(x)=q_{s^{\prime}} \circ f_{n} \circ p_{s^{\prime}}(x)=q_{s} \circ f_{n} \circ p_{s}(x+1)-a, \quad \text { and } \\
& g_{j}^{1}(x)=q_{s} \circ f_{n} \circ p_{s}(x-1)-b .
\end{aligned}
$$

Substituting this into the equation for $h_{r}^{1}$, we have

$$
h_{r}^{1}=q_{s} \circ f_{n} \circ p_{s} \mid I_{0} \cup I_{2} .
$$

We choose now some $r^{\prime}$ such that $f_{s}^{\prime} \in \mathscr{F}_{r r^{\prime}}^{\prime}$ and define, if $s \in K_{n}^{\prime \prime}$,

$$
f_{n}\left|s=q_{s}^{-1} \circ h_{r r}^{1} \circ p_{s}^{-1}\right| s .
$$

Equations (3) and (6) define a PL map $f_{n}: S \rightarrow C$. We show that it has the desired properties.

We first show that $f_{n}$ satisfies (A1). We have $\left\|h_{r r^{\prime}}^{1}(x)-f_{s}^{\prime}(x)\right\|<\varepsilon \leqq 1 / 4 L<1$ for $x \in[-1,2], s \in K_{n}^{\prime \prime}$, when $r$ and $r^{\prime}$ are as in (6). Therefore

$$
\left\|f_{n}(x)-f(x)\right\|<2^{-n} / 4 L \text { for } x \in S,
$$

and we have (A1). To show (A2), let

$$
\varrho=\inf \left\{\left\|h_{i j}^{t}(x)-g(y)\right\|: x, y \in J, g \in \mathscr{F}_{i j}^{\prime}, i \leqq k^{\prime}, j \leqq n_{i}, t=1,2\right\}>0
$$

which depends only on $L$. Then, if $x, y \in s \cup s^{\prime}$ for some adjacent $s, s^{\prime} \in K_{n}$,

$$
\left\|f_{n}(x)-f(y)\right\| \geqq \varrho / 2^{n} .
$$


If this is not true for $x, y \in S$, then $\|x-y\| \geqq 2^{-n}$, and thus

$$
\begin{gathered}
\left\|f_{n}(x)-f(y)\right\| \geqq\|f(x)-f(y)\|-\left\|f_{n}(x)-f(x)\right\| \\
\geqq 2^{-n} / L-2^{-n} / 4 L=(3 / 4 L) 2^{-n}
\end{gathered}
$$

by (7), and (A2) is true if $\delta=\min (3 / 4 L, \varrho)$, which depends only on $L$.

We then show that $f_{n}$ is an embedding. If $x, y \in S, x \neq y$, are in the same simplex of $K_{n}$ or in adjacent simplexes of $K_{n}$, it is clear by the definition of $f_{n}$ that $f_{n}(x) \neq f_{n}(y)$. If this is not the case, $\|x-y\| \geqq 2^{-n}$, and we have

$$
\begin{aligned}
& \left\|f_{n}(x)-f_{n}(y)\right\| \geqq\|f(x)-f(y)\|-\left\|f_{n}(x)-f(x)\right\|-\left\|f_{n}(y)-f(y)\right\| \\
& \geqq 2^{-n} / L-2^{-n-2} / L-2^{-n-2} / L>0,
\end{aligned}
$$

by (7). Therefore $f_{n}$ is an embedding.

It follows directly from the definition of the maps $f_{n}$ that the similarity classes of maps of the form (A3) are in a finite set depending only on $L$. For representatives of the similarity classes we can choose maps of the form $h_{i j}^{t} \mid[0,2]$ and $h_{i j}^{t} \mid[-1,1]$ if $s \cap s^{\prime}$ is not a corner of $S$.

We then consider (A4). If $s \in K_{n}, n \geqq 1$, is a 1 -simplex, let $q_{s}$ be as in (2). The endpoints of $s$ we denote by $u_{s}$ and $v_{s}$. The definition of $f_{n}$ in (3) and (6) implies that there is a finite set $X \subset R^{2}$ depending only on $L$ such that $q_{s}\left(f_{n}\left(u_{s}\right)\right) \in X$ and $q_{s}\left(f_{n}\left(v_{s}\right)\right) \in X$ for all $s \in K_{n}, n \geqq 1$. Let then $s^{\prime} \in K_{n+1}, s^{\prime} \subset s$, be a 1-simplex. Consider the triple

$$
\left(q_{s}\left(f_{n}\left(u_{s}\right)\right), q_{s}\left(f_{n}\left(v_{s}\right)\right), q_{s}\left(f_{n+1}\left(u_{s^{\prime}}\right)\right)\right)=\left(q_{s}\left(f_{n}\left(u_{s}\right)\right), q_{s}\left(f_{n}\left(v_{s}\right)\right), q_{s} \circ q_{s^{\prime}}^{-1} \circ q_{s^{\prime}}\left(f_{n+1}\left(u_{s^{\prime}}\right)\right)\right) .
$$

We have $q_{s} \circ q_{s^{\prime}}^{-1}(x)=x / 2-2^{n}\left(a_{s}-a_{s^{\prime}}\right)$. Since $2^{n}\left(a_{s}-a_{s^{\prime}}\right) \in Z^{2} / 2$ and $\left\|2^{n}\left(a_{s}-a_{s^{\prime}}\right)\right\| \leqq$ $L+2$, the maps $q_{s} \circ q_{s^{\prime}}^{-1}$ vary in a finite set depending only on $L$. Since also $q_{s^{\prime}}\left(f_{n+1}\left(u_{s^{\prime}}\right)\right) \in X$, this implies that triples of the above form are in a finite set depending only on $L$, proving (A4).

Finally, (A5) follows by (7).

The proof of Lemma 2B is similar. Let $L_{n}$ be the triangulation of $I^{1}$ whose 1-simplexes are $I_{n 0}=\left[-2^{-n}, 2^{-n}\right]$ and $I_{n i}=\left[i 2^{-n},(i+1) 2^{-n}\right],-2^{n} \leqq i \leqq-2$, $1 \leqq i \leqq 2^{n}-1$. Let $L_{n}^{\prime}=\left\{I_{n i}: i=-2^{n},-2^{n}+2, \ldots,-2,1,3, \ldots, 2^{n}-1\right\}$ and let $L_{n}^{\prime \prime}=$ $\left\{I_{n i}: i=-2^{n}+1,-2^{n}+3, \ldots,-3,0,2,4, \ldots, 2^{n}-2\right\}$. Then $L_{n}^{\prime} \cup L_{n}^{\prime \prime}$ is the set of 1-simplexes of $L_{n}$, and if $s, s^{\prime} \in L_{n}$ are adjacent, then one of them is in $L_{n}^{\prime}$, the other in $L_{n}^{\prime \prime}$. As above, we first define $f_{n}^{\prime}$ in $\left(\cup L_{n}^{\prime}\right) \times\left\{ \pm 2^{-n}\right\} \cup\{ \pm 1\} \times\left[-2^{-n}, 2^{-n}\right]$ and then in the remaining points. Let $\mathscr{F}=\left\{g: g\right.$ is a $4 L$-embedding $J \rightarrow R^{2}$ with $\|g(0)\| \leqq 1\}$ and $\mathscr{F}^{\prime}=\left\{g: g\right.$ is a $4 L$-embedding $J^{\prime} \rightarrow R^{2}$ with $\left.\|g(0)\| \leqq 1\right\}$. Choose $\varepsilon=\varepsilon(L) \leqq 1 / 16 L$ that satisfies conditions (i)-(iii) of Section 9 with this $\mathscr{F}$ and $\mathscr{F}^{\prime} ; \varepsilon^{\prime} \leqq \varepsilon / 16 L^{2}$ of Lemma 1 (b) is defined with this $\varepsilon$ and $\mathscr{F}^{\prime}$. Now we can define $f_{n}^{\prime} \mid\left(\cup L_{n}^{\prime} \backslash\left(I_{n,-2^{n}} \cup I_{n, 2^{n}-1}\right)\right) \times\left\{ \pm 2^{-n}\right\}$ using Lemma 1 (a) and $f_{n}^{\prime} \mid\left(I_{n,-2^{n}} \cup I_{n, 2^{n}-1}\right) \times$ $\left\{ \pm 2^{-n}\right\} \cup\{ \pm 1\} \times\left[-2^{-n}, 2^{-n}\right]$ using Lemma 1 (c), like $f_{n} \mid \cup K_{n}^{\prime}$ above. Then we extend this to $\left(\cup L_{n}^{\prime \prime}\right) \times\left\{ \pm 2^{-n}\right\}$ as in the proof of Lemma $2 \mathrm{~A}$. This is done in such a way that $f_{n}^{\prime}\left(x, 2^{-n}\right)$ is on the "left" side and $f_{n}^{\prime}\left(x,-2^{-n}\right)$ on the "right" side of 
$f^{\prime}\left(I^{1}\right)$ for $x \in\left[-1+2^{-n}, 1-2^{-n}\right]$. (If $x$ is near the endpoints of $I^{1}$, these relations are not defined.) There are only two points to take care of. The interval $I_{n 0}=$ $\left[-2^{-n}, 2^{-n}\right]$ has twice the length of the other intervals $I_{n i}$ and $f_{n}^{\prime} \mid\left(I_{n,-2^{n}+1} \cup I_{n, 2^{n}-2}\right) \times$ $\left\{ \pm 2^{-n}\right\}$ cannot be defined exactly as above since there is only one 1 -simplex to the left of $I_{n,-2^{n}+1}$ (resp. to the right of $I_{n, 2^{n}-2}$ ). Therefore we must perform some compression and decompression first for $I_{n 0}$ and then for $I_{n,-2^{n}+1}$ and $I_{n, 2^{n}-2}$. (That is why $\mathscr{F}$ and $\mathscr{F}^{\prime}$ are families of $4 L$-embeddings.)

We show that the maps $f_{n}^{\prime}$ turn round points of $f^{\prime}\left(I^{1}\right)$ once in the positive direction; other conditions of Lemma $2 \mathrm{~B}$ are proved like the similar conditions of Lemma $2 \mathrm{~A}$. It suffices to show that $f_{n}^{\prime}$ turns round $f^{\prime}(0)$ once in the positive direction, i.e.

$$
\int_{S_{n}^{\prime}}\left(1 /\left(f_{n}^{\prime}(z)-f^{\prime}(0)\right)\right) d f_{n}^{\prime}(z)=2 \pi i,
$$

where $S_{n}^{\prime}$ has the natural orientation. Let $S_{n}^{0}=\partial I_{n 0}^{2}=\partial\left[-2^{-n}, 2^{-n}\right]^{2}, S_{n}^{+}=\partial\left(\left[2^{-n}, 1\right] \times\right.$ $\left.\left[-2^{-n}, 2^{-n}\right]\right), S_{n}^{-}=\partial\left(\left[-1,-2^{-n}\right] \times\left[-2^{-n}, 2^{-n}\right]\right)$. We extend $f_{n}^{\prime}$ to $S_{n}^{0} \cup S_{n}^{+} \cup S_{n}^{-}$by the requirement that $f_{n}^{\prime} \mid\left\{ \pm 2^{-n}\right\} \times\left[-2^{-n}, 2^{-n}\right]$ is affine. Since $f^{\prime}$ is an $L$-embedding, (B1) implies $f^{\prime}(0) \notin f_{n}^{\prime}\left(S_{n}^{0} \cup S_{n}^{+} \cup S_{n}^{-}\right)$. Therefore the above integral can be written in the form

$$
\int_{S_{n}^{\prime}}=\int_{S_{n}^{0}}+\int_{S_{n}^{+}}+\int_{S_{n}^{-}}
$$

where $S_{n}^{0}, S_{n}^{-}$and $S_{n}^{+}$have the natural orientation. But, using again (B1) and the fact that $f^{\prime}$ is an $L$-embedding, we can deform $f_{n}^{\prime} \mid S_{n}^{+}$and $f_{n}^{\prime} \mid S_{n}^{-}$to constant mappings in $R^{2} \backslash\left\{f^{\prime}(0)\right\}$. Therefore in the above sum the last two integrals vanish and we have

$$
\int_{S_{n}^{\prime}}\left(1 /\left(f_{n}^{\prime}(z)-f^{\prime}(0)\right)\right) d f_{n}^{\prime}(z)=\int_{S_{n}^{0}}\left(1 /\left(f_{n}^{\prime}(z)-f^{\prime}(0)\right)\right) d f_{n}^{\prime}(z) .
$$

But now (iii) of Section 9 implies that the right side integral equals $2 \pi i$. To see this, transform $f^{\prime} \mid I_{n,-2} \cup I_{n 0} \cup I_{n 1}$ and $f_{n}^{\prime} \mid S_{n}^{0}$ as in (2) to get $g_{1}: J \rightarrow R^{2}$ and $g_{2}: S \rightarrow R^{2}$, like the maps $f$ and $g$ of condition (iii) in Section 9. Then the construction of $f_{n}^{\prime}$ and (iii) of Section 9 guarantee that the right side integral equals $2 \pi i$, proving our claim.

11. In the preceding section we showed that, given an $L$-embedding $f: S \rightarrow R^{2}$, there are PL embeddings $f_{i}: S \rightarrow R^{2}, i \geqq 1$, such that $f_{i}(S) \subset C$, where $C$ is the bounded component of $R^{2} \backslash f(S)$. In this section we show that there is $m=m(L)>0$ such that $f_{m}(S), f_{2 m}(S), \ldots$ is a sequence of concentric PL circles converging to $f(S)$ (cf. the right side of Fig. 1) and that there are crossbars which divide the right side of Fig. 1 into a countable family of PL disks. We take care that the number of similarity classes of these PL disks is finite. The construction is illustrated by Fig. 1. Note that we have deleted some crossbars for clarity and that, in view of (A1), the PL circles $f_{i m}(S)$ are closer to $f(S)$ than shown in Fig. 1.

Let $m \in N$ be a number, depending only on $L$, such that $2^{-m}<\delta$, where $\delta$ is as in (A2), and that the following holds: 
(M) Let $g$ be an L-embedding $S \rightarrow R^{2}$ and let $g^{\prime}$ and $g^{\prime \prime}$ be embeddings $S \rightarrow R^{2}$ such that

$$
\left\|g^{\prime}(x)-g(x)\right\| \leqq 2^{-m} \text { and }\left\|g^{\prime \prime}(x)-g(x)\right\| \leqq 2^{-m}
$$

for $x \in S$. If $g^{\prime}(S) \cap g^{\prime \prime}(S)=\emptyset$, then either $g^{\prime}(S)$ is inside $g^{\prime \prime}(S)$ or vice versa (i.e. $g^{\prime}(S)$ is in the bounded component of $R^{2} \backslash g^{\prime \prime}(S)$ or vice versa).

To see the existence of $m=m(L)$ satisfying (M) fix the $L$-embedding $g$ and a point $x$ inside $g(S)$. Then there is $\varepsilon_{g}>0$ such that if $g^{\prime}: S \rightarrow R^{2}$ is an embedding with $\left\|g-g^{\prime}\right\| \leqq \varepsilon_{g}, \quad x$ is inside $g^{\prime}(S)$, too. Now the existence of $m$ follows from this and from the compactness of the set of $L$-embeddings $h: S \rightarrow R^{2}$ with $h(1,1)=0$.

Let $f_{i}, i \geqq 1$, be the maps constructed in Lemma $2 \mathrm{~A}$ and consider the sequence $f_{m}(S), f_{2 m}(S), \ldots$ of PL circles, which are inside $f(S)$. Let $i<j$. By (A1) and (A2), $f_{i m}(S) \cap f_{j m}(S)=\emptyset$. These imply that we can even connect $f_{j m}(S)$ to $f(S)$ by a line segment not touching $f_{i m}(S)$. This and (M) imply that $f_{i m}(S)$ is inside $f_{j m}(S)$. Therefore in the sequence $f_{m}(S), f_{2 m}(S), \ldots$ every circle is inside the next one and all are inside $f(S)$.

Now we define

and let $g_{1}: A \rightarrow R^{2}$ be defined by

$$
A=\bigcup_{i \geqq 1}\left(1-2^{-i m}\right) S
$$

$$
g_{1}\left(\left(1-2^{-i m}\right) x\right)=f_{i m}(x), \quad x \in S, \quad i \geqq 1 .
$$

Then $g_{1}$ is a PL embedding. Let, if $a \in S$ and $n \geqq 1, s_{n}(a)$ be the line segment with endpoints $\left(1-2^{-n m}\right) a$ and $\left(1-2^{-(n+1) m}\right) a$ and let

$$
G=A \cup\left(\cup\left\{s_{n}(a): a \in S \cap Z^{2} / 2^{n m}, n \geqq 1\right\}\right) .
$$

Then $G$ divides int $I^{2}$ into a countable number of quadrilaterals. We denote these by $Q_{0}, Q_{1}, \ldots$ and assume that $Q_{0}$ is the square with boundary $\left(1-2^{-m}\right) S$ and that the quadrilaterals contained in a ring with boundary $\left(1-2^{-i m}\right) S \cup\left(1-2^{-(i+1) m}\right) S$ are successively in the sequence $Q_{0}, Q_{1}, \ldots$. For every $Q_{i}$ we choose a homeomorphism $q_{i}: I^{2} \rightarrow Q_{i}$ that is simplicial in a triangulation of $I^{2}$ with the (four) vertices $( \pm 1, \pm 1)$. Notice that we can express $q_{i}$ in the form

$$
q_{i}(x)=p_{i}\left(\lambda_{i} x\right), \quad x \in I^{2}, \quad i \geqq 0,
$$

where $\lambda_{i}=\operatorname{diam} Q_{i}$ and $p_{i}: \lambda_{i} I^{2} \rightarrow Q_{i}, i \geqq 0$, is a PL $K$-homeomorphism for some $K \geqq 1$ not depending on $L$ nor on $f$. Thus, although the set of the similarity classes of the quadrilaterals $Q_{i}$ is not finite, the distortion from square-form cannot be arbitrary.

Lemma 3A. Let $m=m(L) \in N$ satisfy $(\mathbf{M})$ and assume $2^{-m} \leqq \delta$, where $\delta$ is as in (A2), let $A$ be defined by (8) and let $g_{1}: A \rightarrow R^{2}$ be the embedding defined in (9), where the maps $f_{n}$ are as in Lemma $2 \mathrm{~A}$. Then, if $G$ is as in (10), $g_{1}$ can be extended to a PL embedding $g_{2}: G \rightarrow R^{2}$ in such a way that the similarity classes of the maps $g_{2} \circ\left(q_{i} \mid S\right), i \geqq 0$, are in a finite set depending only on $L$. 
In Lemma 3B we let $m^{\prime}=m^{\prime}(L) \in N$ be a number such that $2^{-m^{\prime}} \leqq \delta^{\prime}$, where $\delta^{\prime}$ is as in (B2), and that:

(M') Conditions (i)-(iii) of Section 9 are true when $\varepsilon=2^{-m^{\prime}}, \mathscr{F}=\left\{g: g: J \rightarrow R^{2}\right.$ an L-embedding, $\|g(0)\| \leqq 1\}$ and $\mathscr{F}^{\prime}=\left\{g: g: J^{\prime} \rightarrow R^{2}\right.$ an L-embedding, $\left.\|g(0)\| \leqq 1\right\}$.

Let $f_{i}^{\prime}: S_{i}^{\prime}=\partial\left(I^{1} \times\left[-2^{-i}, 2^{-i}\right]\right) \rightarrow R^{2}, i \geqq 1$, be the maps constructed in Lemma 2B. Let $C_{k}=f_{k m^{\prime}}^{\prime}\left(S_{k m^{\prime}}^{\prime}\right)$ and let $i<j$. .By (B1) and (B2), $C_{i} \cap C_{j}=\emptyset$. Since we can join $C_{j}$ and $f^{\prime}\left(I^{1}\right)$ by a line segment not touching $C_{i}$ and since $f^{\prime}\left(I^{1}\right)$ is inside both $C_{i}$ and $C_{j}$, we must have that $C_{j}$ is inside $C_{i}$. Therefore in the sequence $f_{m}\left(S_{m^{\prime}}\right), f_{2 m^{\prime}}^{\prime}\left(S_{2 m^{\prime}}^{\prime}\right), \ldots$ every circle is inside the preceding one and $f^{\prime}\left(I^{1}\right)$ is inside all of them.

Let $S_{n}^{\prime \prime}=\partial\left(\left[-1-2^{-n}, 1+2^{-n}\right] \times\left[-2^{-n}, 2^{-n}\right]\right), n \geqq 1$. Define

Let $g_{1}^{\prime}: A^{\prime} \rightarrow R^{2}$ be the map

$$
A^{\prime}=\bigcup_{i \geqq 1} S_{i m^{\prime}}^{\prime \prime}
$$

$$
g_{1}^{\prime}\left(\left(1+2^{-i m^{\prime}}\right) x, y\right)=f_{i m^{\prime}}^{\prime}(x, y), \quad(x, y) \in S_{i m^{\prime}}^{\prime}, i \geqq 1 .
$$

Then $g_{1}^{\prime}$ is a PL embedding. Let, if $a \in I^{1}, n \geqq 1$ and $t= \pm 1, s_{n}^{t}(a)$ be the line segment with endpoints $\left(\left(1+2^{-n m^{\prime}}\right) a, t 2^{-n m^{\prime}}\right)$ and $\left(\left(1+2^{-(n+1) m^{\prime}}\right) a, t 2^{-(n+1) m^{\prime}}\right)$. Let

$$
G^{\prime}=A^{\prime} \cup\left(\cup\left\{s_{n}^{t}(a): a \in I^{1} \cap Z / 2^{n m^{\prime}}, t= \pm 1, n \geqq 1\right\}\right) .
$$

Then $G^{\prime}$ divides $\left[-1-2^{-m^{\prime}}, 1+2^{-m^{\prime}}\right] \times\left[-2^{-m^{\prime}}, 2^{-m^{\prime}}\right] \backslash I^{1}$ into a countable number of quadrilaterals, denoted $Q_{0}^{\prime}, Q_{1}^{\prime}, \ldots$. As above, we can find PL homeomorphisms $q_{i}^{\prime}: I^{2} \rightarrow Q_{i}^{\prime}$ and $p_{i}^{\prime}:\left(\operatorname{diam} Q_{i}^{\prime}\right) I^{2} \rightarrow Q_{i}^{\prime}$ such that

$$
q_{i}^{\prime}(x)=p_{i}^{\prime}\left(\left(\operatorname{diam} Q_{i}^{\prime}\right) x\right), \quad x \in I^{2} \quad \text { and } \quad i \geqq 0,
$$

that $q_{i}^{\prime}$ is simplicial in a triangulation of $I^{2}$ with vertices $( \pm 1, \pm 1)$ and that $p_{i}^{\prime}$, $i \geqq 0$, is a $K^{\prime}$-homeomorphism, where $K^{\prime}$ does not depend on $L$ nor on $f^{\prime}$.

Lemma 3B. Let $m^{\prime}=m^{\prime}(L)$ satisfy $\left(\mathrm{M}^{\prime}\right)$ and assume $2^{-m^{\prime}} \leqq \delta^{\prime}$, where $\delta^{\prime}$ is as in (B2), let $A^{\prime}$ be defined by ( $\left.8^{\prime}\right)$ and let $g_{1}^{\prime}: A^{\prime} \rightarrow R^{2}$ be the embedding defined in $\left(9^{\prime}\right)$, where the maps $f_{n}^{\prime}$ are as in Lemma $2 \mathrm{~B}$. Then if $G^{\prime}$ is as in $\left(10^{\prime}\right), g_{1}^{\prime}$ can be extended to a PL embedding $g_{2}^{\prime}: G^{\prime} \rightarrow R^{2}$ in such a way that the similarity classes of the maps $g_{2}^{\prime} \circ\left(q_{i}^{\prime} \mid S\right), i \geqq 0$, are in a finite set depending only on $L$.

Proof. We consider first Lemma 3A. Let $R_{n}$ be the component of

$$
R^{2} \backslash\left(f_{n m}(S) \cup f_{(n+1) m}(S)\right)
$$

with boundary $f_{n m}(S) \cup f_{(n+1) m}(S)$. Then $R_{n} \cap f(S)=\emptyset$.

If $x \in S$, let $r_{n}(x)$ be the line segment joining $f_{n m}(x)$ and $f_{(n+1) m}(x)$. By (A1) $r_{n}(x) \subset U_{\varepsilon}(f(x))$, where $\varepsilon=2^{-n m} / 4 L$. Thus

$$
r_{n}(x) \cap r_{n}(y)=\emptyset \quad \text { if } \quad x, y \in S \text { and }\|x-y\| \geqq 2^{-n m} .
$$


Therefore the line segments $r_{n}(a), a \in S \cap 2^{-n m} Z^{2}$, form a disjoint family for fixed $n$. Let $r_{n}^{\prime}(a)$ be a subsegment of $r_{n}(a)$ such that int $r_{n}^{\prime}(a) \subset R_{n}, r_{n}^{\prime}(a) \cap f_{n m}(S)=\left\{f_{n m}\left(x_{n a}\right)\right\}$ and $r_{n}^{\prime}(a) \cap f_{(n+1) m}(S)=\left\{f_{(n+1) m}\left(y_{n a}\right)\right\}$, where $x_{n a}$ and $y_{n a} \in S$. Now we can find a $\mathrm{PL}$ arc $r_{n}^{\prime \prime}(a)$ joining $f_{n m}(a)$ and $f_{(n+1) m}(a)$ such that int $r_{n}^{\prime \prime}(a) \subset R_{n}$. This can be constructed from a subsegment of $r_{n}^{\prime}(a)$ and from PL arcs close to the arcs $f_{n m}\left(x_{n a} a\right)$ and $f_{(n+1) m}\left(y_{n a} a\right)$, where $x_{n a} a$ and $y_{n a} a$ are certain subarcs of $S$ with endpoints $\left\{x_{n a}, a\right\}$ and $\left\{y_{n a}, a\right\}$. Since $\left\|f_{n m}\left(x_{n a}\right)-f(a)\right\|<2^{-n m} / 4 L,\left\|f\left(x_{n a}\right)-f(a)\right\|<2^{-n m} / 4 L+$ $\left\|f_{n m}\left(x_{n a}\right)-f\left(x_{n a}\right)\right\|<2^{-n m} / 2 L$ by (A1). Therefore $\left\|x_{n a}-a\right\|<2^{-n m} / 2$, since $f$ is an $L$-embedding. Hence $x_{n a} a$ is a line segment and, if $b \in S \cap 2^{-n m} Z^{2}, b \neq a$, we have $\|x-b\|>2^{-n m} / 2$ for every $x \in x_{n a} a$, implying that

$$
\left\|f_{n m}(x)-f(b)\right\| \geqq\|f(x)-f(b)\|-\left\|f_{n m}(x)-f(x)\right\|>2^{-n m} / 2 L-2^{-n m} / 4 L=2^{-n m} / 4 L .
$$

Therefore $f_{n m}\left(x_{n a} a\right) \cap r_{n}^{\prime}(b)=\emptyset$ and, similarly, $f_{(n+1) m}\left(y_{n a} a\right) \cap r_{n}^{\prime}(b)=\emptyset$, and, since $x_{n a} a \cap x_{n b} b=y_{n a} a \cap y_{n b} b=\emptyset$, we can assume that, for every $n \geqq 1$, the family $\left\{r_{n}^{\prime \prime}(a): a \in S \cap 2^{-n m} Z^{2}\right\}$ is a disjoint family of PL arcs.

Now we can extend $g_{1}$ to a PL embedding $g_{2}: G \rightarrow R^{2}$ in such a way that $g_{2}\left(s_{n}(a)\right)=r_{n}^{\prime \prime}(a)$ for $a \in S \cap 2^{-n m} Z^{2}$. It follows from (A4) that the similarity classes of the quadrilaterals with vertices $\left\{f_{n m}(u), f_{n m}(v), f_{(n+1) m}(u), f_{(n+1) m}(v)\right\}, n \geqq 1$, where $u$ and $v$ are the endpoints of a 1-simplex of $K_{n m}$, are in a finite set depending only on $L$. Using this and (A3) we can easily see that we can choose the $\operatorname{arcs} r_{n}^{\prime \prime}(a)$ and the extension of $g_{1}$ to the segments $s_{n}(a)$ in such a way that the similarity classes of the maps $g_{2} \circ\left(q_{i} \mid S\right)$ are in a finite set depending only on $L$.

Now we turn to the proof of Lemma 3B. For $n \geqq 1$ let $s_{n k}=\left[k 2^{-n m^{\prime}},(k+1) 2^{-n m^{\prime}}\right]$, $-2^{n m^{\prime}} \leqq k<2^{n m^{\prime}}$. Let $U_{n k}=\cup\left\{U_{2-n m^{\prime}-m^{\prime} / 4 L}\left(f^{\prime}(x)\right): x \in s_{n k}\right\} \backslash \operatorname{im} f^{\prime}$. Then in view of $\left(\mathrm{M}^{\prime}\right)$ and (ii) of Section 9, $U_{n k},-2^{n m^{\prime}}<k<2^{n m^{\prime}}-1$, has exactly two components $C_{n k}^{1}$ and $C_{n k}^{2}$ such that $f^{\prime}\left(s_{n k}\right) \subset \operatorname{cl} C_{n k}^{t}, t=1,2$. Let the notation $C_{n k}^{1}$ and $C_{n k}^{2}$ be as in (iii) of Section 9, i.e. $C_{n k}^{1}$ is on the "left" side of $f^{\prime}$. Let $J_{n}=\left[-1+2^{-n m^{\prime}}, 1-2^{-n m^{\prime}}\right]$ and let

$$
U_{n}=\cup\left\{U_{2^{-n m^{\prime}-m^{\prime} / 4 L}}\left(f^{\prime}(x)\right): x \in J_{n}\right\} \backslash \operatorname{im} f^{\prime}=\cup\left\{U_{n k}:-2^{n m^{\prime}}<k<2^{n m^{\prime}}-1\right\} .
$$

Let $C_{n}^{t}=\cup\left\{C_{n k}^{t}:-2^{n m^{\prime}}<k<2^{n m^{\prime}}-1\right\}, t=1,2$. Then by (ii) of Section $9, C_{n}^{1}$ and $C_{n}^{2}$ are the two components of $U_{n}$ such that $f^{\prime}\left(J_{n}\right) \subset \operatorname{cl~} C_{n}^{t}, t=1,2$. By (B1) $f_{n m^{\prime}}^{\prime}\left(0, \pm 2^{-n m^{\prime}}\right) \in C_{n-1}^{1} \cup C_{n-1}^{2}$ if $n>1$ (we can join $f_{n m^{\prime}}^{\prime}\left(0, \pm 2^{-n m^{\prime}}\right.$ ) by a path $s$ to $f^{\prime}\left(J_{n-1}\right)$ with int $\left.s \subset U_{n-1}\right)$. Therefore either $f_{n m^{\prime}}^{\prime}\left(J_{n-1} \times\left\{t 2^{-n m^{\prime}}\right\}\right) \subset C_{n-1}^{1}$ or $f_{n m^{\prime}}^{\prime}\left(J_{n-1} \times\left\{t 2^{-n m^{\prime}}\right\}\right) \subset C_{n-1}^{2}, t= \pm 1$. We show that

$$
f_{n m^{\prime}}^{\prime}\left(J_{n-1} \times\left\{2^{-n m^{\prime}}\right\}\right) \subset C_{n-1}^{1} \text { and } f_{n m^{\prime}}^{\prime}\left(J_{n-1} \times\left\{-2^{-n m^{\prime}}\right\}\right) \subset C_{n-1}^{2} .
$$

Let $\bar{S}_{n m^{\prime}}=\partial\left(\left[0,2^{-(n-1) m^{\prime}}\right] \times\left[-2^{-n m^{\prime}}, 2^{-n m^{\prime}}\right]\right)$ and extend $f_{n m^{\prime}}^{\prime}$ to $\bar{S}_{n m^{\prime}} \cup S_{n m^{\prime}}^{\prime}$ by the requirement that the maps $f_{n m^{\prime}}^{\prime} \mid\{x\} \times\left[-2^{-n m^{\prime}}, 2^{-n m^{\prime}}\right], x \in\left\{0,2^{-(n-1) m^{\prime}}\right\}$, are affine. Then $f^{\prime}\left(2^{-(n-1) m^{\prime}} / 2\right) \notin \operatorname{im} f_{n m^{\prime}}^{\prime}$ and one sees exactly as in the last paragraph of Section 10 that both $f_{n m^{\prime}}^{\prime} \mid S_{n m^{\prime}}^{\prime}$ and $f_{n m^{\prime}}^{\prime} \mid \bar{S}_{n m^{\prime}}$ turn around $f^{\prime}\left(2^{-(n-1) m^{\prime}} / 2\right) N^{\prime}$ times, $N^{\prime} \in Z$, when $S_{n m^{\prime}}^{\prime}$ and $\bar{S}_{n m^{\prime}}$ both have the natural orientation. But for $f_{n m^{\prime}}^{\prime} \mid S_{n m^{\prime}}^{\prime}$ 
we know by assumption that $N^{\prime}=1$. Now we can apply (iii) of Section 9 (after blowing up the maps $f^{\prime} \mid\left[-2^{-(n-1) m^{\prime}}, 2^{-(n-1) m^{\prime}} \cdot 2\right]$ and $f_{n m^{\prime}}^{\prime} \mid \bar{S}_{n m^{\prime}}$ in such a way that they become maps $J \rightarrow R^{2}$ and $\left.S \rightarrow R^{2}\right)$ and get the result that $f_{n m^{\prime}}^{\prime}\left(x, 2^{-n m^{\prime}}\right) \in C_{n-1,0}^{1} \subset$ $C_{n-1}^{1}$ and $f_{n m^{\prime}}^{\prime}\left(x,-2^{-n m^{\prime}}\right) \in C_{n-1,0}^{2} \subset C_{n-1}^{2}$, where $x=2^{-(n-1) m^{\prime}} / 2$. This implies (12).

Let $a \in J_{n-1}$ and let $r_{n}^{t}(a), t= \pm 1$, be the line segment with endpoints $f_{n m^{\prime}}^{\prime}\left(a, t 2^{-n m^{\prime}}\right)$ and $f_{(n+1) m^{\prime}}^{\prime}\left(a, t 2^{-(n+1) m^{\prime}}\right)$. By (B1) $r_{n}^{1}(a) \subset U_{2^{-n m^{\prime}} / 4 L}\left(f^{\prime}(a)\right)$ and by (12) $f_{n m^{\prime}}^{\prime}\left(a, 2^{-n m^{\prime}}\right) \in C_{n-1}^{1}$. Therefore there is a maximal half-open or closed subinterval $\bar{r}_{n}^{1}(a) \subset C_{n-1}^{1}$ of $r_{n}^{1}(a)$ such that one endpoint of $\bar{r}_{n}^{1}(a)$ is $f_{n m^{\prime}}^{\prime}\left(a, 2^{-n m^{\prime}}\right)$, the other endpoint lies on $f^{\prime}\left(I^{1}\right)$ or is $f_{(n+1) m^{\prime}}^{\prime}\left(a, 2^{-(n+1) m^{\prime}}\right)$. Since $f^{\prime}\left(I^{1}\right)$ is inside $f_{(n+1) m^{\prime}}^{\prime}\left(S_{(n+1) m^{\prime}}^{\prime}\right)$, which is inside $f_{n m^{\prime}}^{\prime}\left(S_{n m^{\prime}}^{\prime}\right)$, we can find in both cases a subsegment $r_{n}^{\prime \prime}(a) \subset C_{n-1}^{1}$ of $\bar{r}_{n}^{1}(a)$ such that int $r_{n}^{\prime \prime}(a) \cap\left(f_{n m^{\prime}}^{\prime}\left(S_{n m^{\prime}}^{\prime}\right) \cup f_{(n+1) m^{\prime}}^{\prime}\left(S_{(n+1) m^{\prime}}^{\prime}\right)\right)=\emptyset$, that one endpoint lies on $f_{n m^{\prime}}^{\prime}\left(S_{n m^{\prime}}^{\prime}\right)$ and the other on $f_{(n+1) m^{\prime}}^{\prime}\left(S_{(n+1) m^{\prime}}^{\prime}\right)$. One defines $r_{n}^{\prime-1}(a) \subset$ $C_{n-1}^{2}$ similarly.

Now one defines the PL arcs $r_{n}^{\prime \prime t}(a), t= \pm 1$, for $a \in 2^{-n m^{\prime}} Z \cap J_{n-1}$ exactly as the arcs $r_{n}^{\prime \prime}(a)$ earlier if $n>1$. We must define $r_{n}^{\prime \prime t}(a)$ also if $n=1$ and $a \in 2^{-m^{\prime}} Z \cap I^{1}$ or if $n>1$ and $a \in 2^{-n m^{\prime}} Z \cap\left(I^{1} \backslash J_{n-1}\right)$. If $n=1$, by (B3) and (B4) the similarity classes of the maps $g_{1}^{\prime} \mid S_{m^{\prime}}^{\prime \prime} \cup S_{2 m^{\prime}}^{\prime \prime}$ are in a finite set depending only on $L$ when $f^{\prime}$ varies in the set of $L$-embeddings $I^{1} \rightarrow R^{2}$. Therefore since the PL annulus theorem is true for $n=2$, and since $f_{m}^{\prime}$ and $f_{2 m}^{\prime}$ are oriented similarly, we can choose separately for each similarity class the $\operatorname{arcs} r_{1}^{\prime \prime t}(a)$. The situation is similar if $a \in 2^{-n m^{\prime}} Z \cap\left(I^{1} \backslash J_{n-1}\right)$. For instance, let $a>0$. We can assume that the similarity classes of the PL Jordan curves

$$
\begin{gathered}
r_{n}^{\prime \prime 1}\left(1-2^{-(n-1) m^{\prime}}\right) \cup r_{n}^{\prime \prime-1}\left(1-2^{-(n-1) m^{\prime}}\right) \\
\cup f_{n m^{\prime}}^{\prime}\left(\left[1-2^{-(n-1) m^{\prime}}, 1\right] \times\left\{ \pm 2^{-n m^{\prime}}\right\} \cup\{1\} \times\left[-2^{-n m^{\prime}}, 2^{-n m^{\prime}}\right]\right) \\
\cup f^{\prime}{ }_{(n+1) m^{\prime}}\left(\left[1-2^{-(n-1) m^{\prime}}, 1\right] \times\left\{ \pm 2^{-(n+1) m^{\prime}}\right\} \cup\{1\} \times\left[-2^{-(n+1) m^{\prime}}, 2^{-(n+1) m}\right]\right)
\end{gathered}
$$

are in a finite set depending only on $L$ when $f$ varies. Therefore the same reasoning applies as in the case $n=1$.

As in the proof of Lemma 3A we conclude, by aid of (B3) and (B4), that we can choose the arcs $r_{n}^{\prime \prime t}(a)$ and the extension $g_{2}^{\prime}$ in such a way that the similarity classes of the maps $g_{2}^{\prime} \circ\left(q_{i}^{\prime} \mid S\right)$ are in a finite set depending only on $L$.

12. After the preceding lemmata the proof of Theorems A and B is not difficult.

Let $C$ be the bounded component of $R^{2} \backslash f(S)$. We first construct an embedding $F^{\prime}: I^{2} \rightarrow \mathrm{cl} C$ extending $f$ and after that extend $F^{\prime}$ to $R^{2}$ as required in Theorem A. Let $g_{2}: G \rightarrow R^{2}$ be the embedding constructed in Lemma 3A. Let $h_{i}: S \rightarrow R^{2}, i \leqq M$, be PL embeddings, depending only on $L$, such that every $g_{2} \circ\left(q_{j} \mid S\right), j \geqq 0$, is similar to some $h_{i}$. Since the PL Schönflies theorem is true for $n=2$, each $h_{i}$ can be extended to a PL embedding $H_{i}: I^{2} \rightarrow R^{2}$. We set now

$$
\begin{aligned}
& F^{\prime} \mid Q_{i}=A_{i} \circ H_{n_{i}} \circ q_{i}^{-1}, \quad i \geqq 0, \quad \text { and } \\
& F^{\prime} \mid S=f .
\end{aligned}
$$


In (13) $A_{i}$ is a similarity of $R^{2}$ and $n_{i} \leqq M$; these are determined in such a way that $F^{\prime}$ extends $g_{2}$. Actually in (13) we should write $B_{i} \circ q_{i}^{-1}$ instead of $q_{i}^{-1}$, where $B_{i}$ is a similarity $I^{2} \rightarrow I^{2}$. But we can redefine $q_{i}$ in such a way that $B_{i}=\mathrm{id}$.

Clearly, $F^{\prime}$ is a bijection $I^{2} \rightarrow \operatorname{cl} C$. We will show:

$1^{\circ}$. For every $i \geqq 0, F^{\prime} \mid Q_{i}$ is an $L_{1}$-embedding, where $L_{1} \geqq L$ depends only on $L$.

$2^{\circ}$. $F^{\prime}$ is $L_{1}$-Lipschitz.

$3^{\circ}$. $F^{\prime-1}$ is $L_{1}$-Lipschitz.

These imply that $F^{\prime}$ is an $L_{1}$-embedding.

To prove $1^{\circ}$ we write (13) in the form

$$
F^{\prime}(x)=A_{i}\left(H_{n_{i}}\left(\lambda_{i}^{-1} p_{i}^{-1}(x)\right)\right), \quad x \in Q_{i},
$$

where (cf. (11), Section 11) $\lambda_{i}=\operatorname{diam} Q_{i}$ and $p_{i}: \lambda_{i} I^{2} \rightarrow Q_{i}$ is a PL $K$-homeomorphism and $K$ does not depend on anything. Now, consider the maps $r_{i}: \lambda_{i} I^{2} \rightarrow R^{2}$, $i \geqq 0$, defined by

$$
r_{i}(x)=A_{i}\left(H_{n_{i}}\left(\lambda_{i}^{-1} x\right)\right), \quad x \in \lambda_{i} I^{2},
$$

which are simlilar to the maps $H_{i}, i \leqq M$. It suffices to show that the maps $r_{i}, i \geqq 0$, are $L_{0}$-embeddings, where $L_{0}$ depends only on $L$. By (A5) we can find two different corner points $x, y \in Q_{i} \cap\left(1-2^{-n}\right) S$, for some $n \geqq 1$, such that

$$
1 / 2 \leqq\left\|r_{i}\left(p_{i}^{-1}(x)\right)-r_{i}\left(p_{i}^{-1}(y)\right)\right\| /\left\|f\left(\left(1-2^{-n}\right)^{-1} x\right)-f\left(\left(1-2^{-n}\right)^{-1} y\right)\right\| \leqq 2
$$

since $r_{i}\left(p_{i}^{-1}(x)\right)=f_{n}\left(\left(1-2^{-n}\right)^{-1} x\right)$ and $r_{i}\left(p_{i}^{-1}(y)\right)=f_{n}\left(\left(1-2^{-n}\right)^{-1} y\right)$. Now, there is an absolute constant $c \geqq 1$ such that $1 / c \leqq\left(1-2^{-n}\right)^{-1}\|x-y\| /\left\|p_{i}^{-1}(x)-p_{i}^{-1}(y)\right\| \leqq c$. Since $f$ is an $L$-embedding, it follows that

$$
1 / 2 c L \leqq\left\|r_{i}\left(p_{i}^{-1}(x)\right)-r_{i}\left(p_{i}^{-1}(y)\right)\right\|||\left\|p_{i}^{-1}(x)-p_{i}^{-1}(y)\right\| \leqq 2 c L .
$$

Since the set of maps $H_{i}, i \leqq M$, depends only on $L$, this implies that we can find such $L_{0}$. This proves $1^{\circ}$.

Since int $I^{2}$ is convex, it follows from $1^{\circ}$ that $F^{\prime}$ int $I^{2}$ is $L_{1}$-Lipschitz. This fact and (A1) imply that $F^{\prime}$ is continuous also on the boundary $S$ of $I^{2}$. It follows that $F^{\prime}$ is $L_{1}$-Lipschitz. That is, we have $2^{\circ}$.

To prove $3^{\circ}$, we choose two points $x, y \in F^{\prime}\left(I^{2}\right), x \neq y$. Let $s$ be the line segment joir.ing $x$ and $y$. If $s \subset F\left(\right.$ int $I^{2}$ ), we have by $1^{\circ}$

$$
\left\|F^{\prime-1}(x)-F^{\prime-1}(y)\right\| \leqq L_{1}\|x-y\|
$$

since we can divide $s$ into subintervals whose endpoints lie in some set $F^{\prime}\left(Q_{i}\right)$, $i \geqq 0$. Notice that (14) is also valid if $x$ or $y \in F^{\prime}(S)$ but the interior points of $s$ are in $F^{\prime}$ (int $I^{2}$ ). If $s \notin F^{\prime}$ (int $I^{2}$ ), let $s_{x}$ be the subinterval of $s$ such that $x \in s_{x}$ and that $s_{x} \cap F(S)$ is an endpoint $x^{\prime}$ of $s_{x}\left(=x\right.$ if $\left.x \in F^{\prime}(S)\right)$. Define similarly $s_{y}$ and $y^{\prime}$. Then (14) is true for the pairs $\left(x, x^{\prime}\right),\left(y, y^{\prime}\right)$ and also for $\left(x^{\prime}, y^{\prime}\right)$ since $F^{\prime} \mid S=f$ is an $L$-embedding. This implies $3^{\circ}$. 
We then extend $F^{\prime}$ to $R^{2}$. We can assume that $F^{\prime}$ is orientation preserving. Replacing $f$ by the map $x \mapsto f(x)-F^{\prime}(0)$ we can assume that $F^{\prime}(0)=0$. Let $r$ be a PL reflection of $\mathrm{cl}\left(\sqrt{2} L_{1} I^{2} \backslash\left(\sqrt{2} L_{1}\right)^{-1} I^{2}\right)$ onto itself such that $r \mid S=\mathrm{id}, r\left(\sqrt{2} L_{1} S\right)=$ $\left(\sqrt{2} L_{1}\right)^{-1} S$. Then $r$ is an $L_{2}$-embedding, $L_{2}=L_{2}(L)$. Consider the $L_{2} L$-embedding $r \circ f: S \rightarrow R^{2}$. Let $C^{\prime}$ be the bounded component of $R^{2} \backslash r(f(S))$. Then we apply the above construction and find an $L_{3}$-embedding $F^{\prime \prime}: I^{2} \rightarrow \mathrm{cl} C^{\prime}$ extending $r \circ f$, where $L_{3}$ depends only on $L$. It is also orientation preserving. Since $r(f(S)) \subset$ $\operatorname{cl}\left(\sqrt{2} L_{1} I^{2} \backslash\left(\sqrt{2} L_{1}\right)^{-1} I^{2}\right),\left(\sqrt{2} L_{1}\right)^{-1} I^{2} \subset \mathrm{cl} C^{\prime}$. Therefore there is a number $k=k(L)$ such that $F^{\prime \prime}\left(\left(1-2^{-k}\right) I^{2}\right) \supset\left\{0, F^{\prime \prime}(0)\right\}$. Now, by the construction of $F^{\prime \prime}$, the similarity classes of the maps $F^{\prime \prime} \mid\left(1-2^{-k-1}\right) I^{2}$ are in a finite set depending only on $L$, when $f$ varies in the set of $L$-embeddings $S \rightarrow R^{2}$. Therefore there is a number $c=c(L)>0$ and a PL map $G: R^{2} \rightarrow R^{2}$ such that $G \circ F^{\prime \prime}\left|c I^{2}=\mathrm{id}, G\right| R^{2} \backslash F^{\prime \prime}\left(\left(1-2^{-k-1}\right) I^{2}\right)=\mathrm{id}$ and that $G \circ F^{\prime \prime}$ is an $L_{4}$-embedding, where $L_{4}$ depends only on $L$. Theferore, if we replace $F^{\prime \prime}$ by $G \circ F^{\prime \prime}$ and $L_{3}$ by $L_{4}$ we can assume $F^{\prime \prime} \mid c I^{2}=$ id.

Let $r^{\prime}$ be an extension of $r$ to a PL reflection of $\mathrm{cl}\left(c^{-1} I^{2} \backslash c I^{2}\right)$ (we assume that $\left.c \leqq 1 / \sqrt{2} L_{1}\right)$. Then $r^{\prime}$ is an $L_{5}$-embedding, where $L_{5}$ depends only on $L$. We define $F: R^{2} \rightarrow R^{2}$ by

$$
F(x)=\left\{\begin{array}{l}
F^{\prime}(x) \text { if } x \in I^{2}, \\
r^{\prime} \circ F^{\prime \prime} \circ r^{\prime}(x) \text { if } \quad x \in c^{-1} I^{2} \backslash I^{2}, \\
x \text { if } x \in R^{2} \backslash c^{-1} I^{2}
\end{array}\right.
$$

this is a homeomorphism extending $f$. Obviously, every one of the maps $F \mid I^{2}$, $F \mid c^{-1} I^{2} \backslash I^{2}$ and $F \mid R^{2} \backslash c^{-1} I^{2}$ is an $L_{6}$-embedding, where $L_{6}=L^{\prime}$ depends only on $L$. But then this is true also for $F$, concluding the proof of Theorem A.

The proof of Theorem B is similar to the above proof. Replacing Lemma 3A by Lemma 3B, (A1) by (B1) and (A5) by (B5) we get an embedding

$$
F_{1}^{\prime \prime}:\left[-1-2^{-m^{\prime}}, 1+2^{-m^{\prime}}\right] \times\left[-2^{-m^{\prime}}, 2^{-m^{\prime}}\right]=A_{m^{\prime}} \rightarrow R^{2} .
$$

The biggest difference is that, if $Q_{i}^{\prime}$ is a quadrilateral with two sides of the from $\left\{ \pm\left(1+2^{-n m^{\prime}}\right)\right\} \times\left[-2^{-n m^{\prime}}, 2^{-n m^{\prime}}\right]$, we cannot conclude directly by (B5) that $F_{1}^{\prime \prime} \mid Q_{i}^{\prime}$ is an $L_{1}^{\prime}(L)$-embedding. However, $Q_{i}^{\prime}$ is adjacent to a quadrilateral $Q_{j}^{\prime}$, of which we can show as above that $F_{1}^{\prime \prime} \mid Q_{j}^{\prime}$ is an $L_{1}^{\prime}(L)$-embedding. Now we can use this fact in place of (B5) to conclude that $F_{1}^{\prime \prime} \mid Q_{i}^{\prime}$ is an $L_{2}^{\prime}(L)$-embedding. Now, if $f^{\prime}$ varies in the set of $L$-embeddings $I^{1} \rightarrow R^{2}$, the similarity classes of the maps $F_{1}^{\prime \prime} \mid \partial A_{m^{\prime}}$ vary in a finite set depending only on $L$. Therefore we can extend $F_{1}^{\prime \prime}$ to a homeomorphism $F^{\prime \prime}$ of $R^{2}$ in such a way that $F^{\prime \prime} \mid \mathrm{cl}\left(R^{2} \backslash A_{m^{\prime}}\right)$ is a PL Lipschitz embedding and that the similarity classes of the maps $F^{\prime \prime} \mid \mathrm{cl}\left(R^{2} \backslash A_{m^{\prime}}\right)$ are in a finite set depending only on $L$. Choose $Q_{i}^{\prime}$ with $Q_{i}^{\prime} \cap \partial A_{m^{\prime}} \neq \emptyset$. Then $F^{\prime \prime} \mid Q_{i}^{\prime}$ is a $\max \left(L_{1}^{\prime}(L), L_{2}^{\prime}(L)\right)$-embedding, implying that $F^{\prime \prime} \mid \mathrm{cl}\left(R^{2} \backslash A_{m^{\prime}}\right)$ is an $L_{3}^{\prime}(L)$-embedding. It follows that $F^{\prime \prime}$ is an $L_{4}^{\prime}(L)$-embedding. Otherwise we omit the proof of Theorem B. 
As a final remark we note that the above proof shows that there is a triangulation $K$ of $R^{2} \backslash S$, depending only on $L$, such that the extension $F$ of $f$ constructed above has the property that $F \mid R^{2} \backslash S$ is simplicial with respect to $K$. A similar remark can be made on Theorem $B$.

\section{Counter-examples and conjectures}

13. We consider now the situation in $R^{3}$, where we can think of generalizations of Theorems A and B both to embeddings of arcs and spheres. Since there are Lipschitz embeddings of arcs and spheres in $R^{3}$ that are not topologically flat (see Luukkainen-Väisälä $[6,3.10]$ ), direct generalizations of Theorems A and B cannot be true. However, one could conjecture that if such an embedding is topologically flat, then it must be also Lipschitz flat (cf. Problem 4 of $[6,9.1]$ ). We show that this conjecture is not true for Lipschitz embeddings of arcs or spheres into $R^{3}$. Finally, we modify this conjecture in such a way that it is reasonable to expect that this conjecture (in $R^{3}$ ) could be proved like the results of this paper, by aid of known result of three-dimensional PL topology.

14. Our construction of a Lipschitz arc which is topologically flat but not locally Lipschitz flat is based on the Fox-Artin arc. Fig. 2 (ignore the three auxi izry broken lines) consists of two Fox-Artin arcs that are joined in the bad point in such a way that the second arc traces backwards, from the bad point to the end, the movements of the first arc from the beginning to the bad point. We denote this arc by $J$. Then $J$ is not locally topologically flat at the bad point. We can assume that there is a Lipschitz homeomorphism $f: I^{1} \rightarrow J$. Next, we form the arcs $J_{n}$, $n>0$, from $J$ by replacing a neighbourhood (in $J$ ) of the bad point by a line segment. These line segments are the broken lines of Fig. 2. There are homeomorphisms $f_{n}: I^{1} \rightarrow J_{n}$ such that $f_{n} \rightarrow f$ as $n \rightarrow \infty$, and that $f$ and $f_{n}, n>0$, are $L$-homeomorphisms for some $L \geqq 1$. In addition, each $J_{n}$ is topologically flat.

Using the arcs $J_{n}$ we form the arc $J_{0}$ of Fig. 3. We take a line segment, remove from it subsegments converging to an interior point $X$, and in place of the removed line segments insert arcs similar to the $\operatorname{arcs} J_{n}, n \geqq 1$. We can do this is such a way that we obtain a Lipschitz arc, parametrized by a Lipschitz embedding $g: I^{1} \rightarrow R^{3}$ such that for each $n>0$ there are similari.ies $g_{n}^{\prime}$ and $g_{n}^{\prime \prime}$ of $R^{3}$ with $f_{n}=$ $g_{n}^{\prime} \circ g \circ\left(g_{n}^{\prime \prime} \mid I^{1}\right)$. The arc $J_{0}$ is topologically flat. To see this, cover the inserted arcs by disjoint 3-balls $B_{i}, i \geqq 1$, and map the portion of $J_{0}$ inside $B_{i}$ onto a line segment by a homeomorphism of $B_{i}$ that fixes $\partial B_{i}$. These maps, extended by the identity outside the balls $B_{i}$, define together a homeomorphism of $R^{3}$ which maps $J_{0}$ onto a line segment.

But $J_{0}$ cannot be locally Lipschitz flat at $X$. Suppose it were. Then let $U$ be a neighbourhood of $X$ in $R^{3}$ and let $F: U_{\lambda}(0) \rightarrow U$ be a Lipschitz homeomorphism with $F((-\lambda, \lambda))=U \cap J_{0}$, where $\lambda>0$. Using this $F$ and the similari.ies $g_{n}^{\prime}$ and 


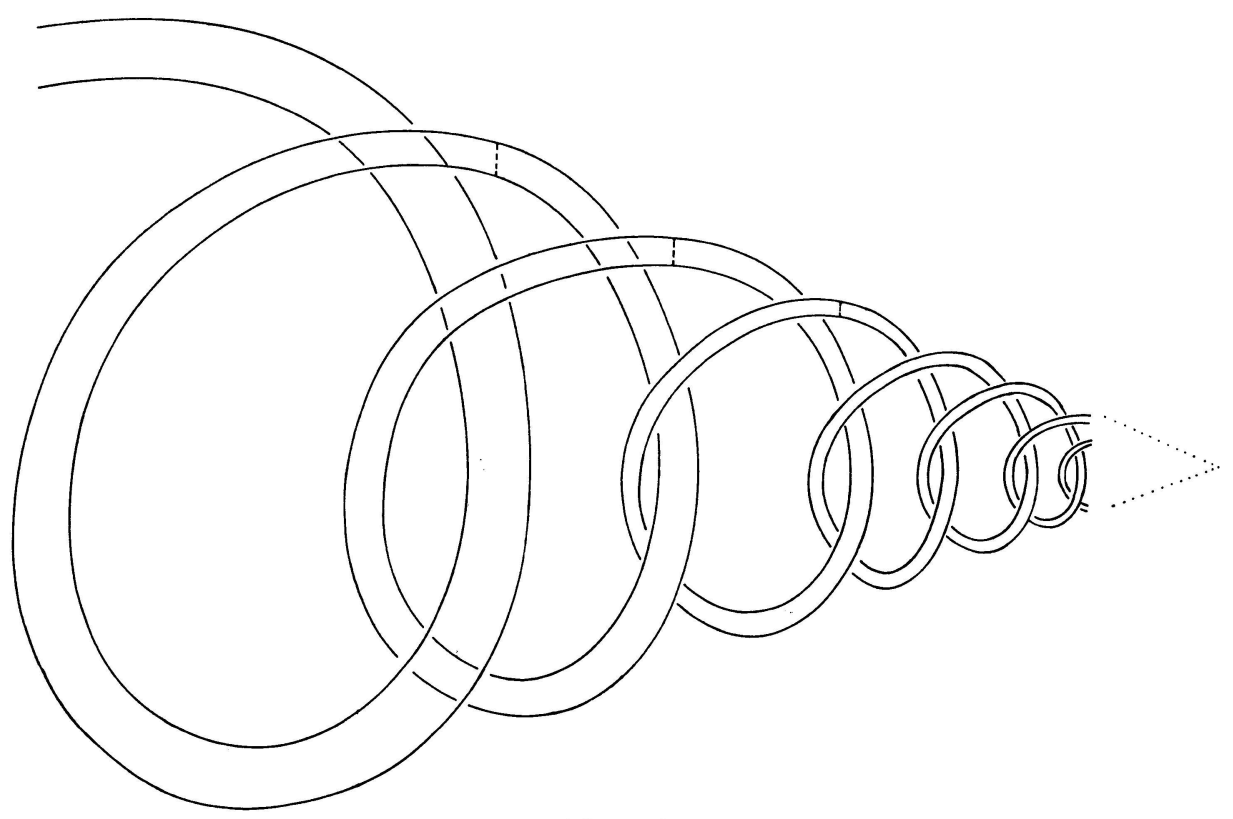

Figure 2
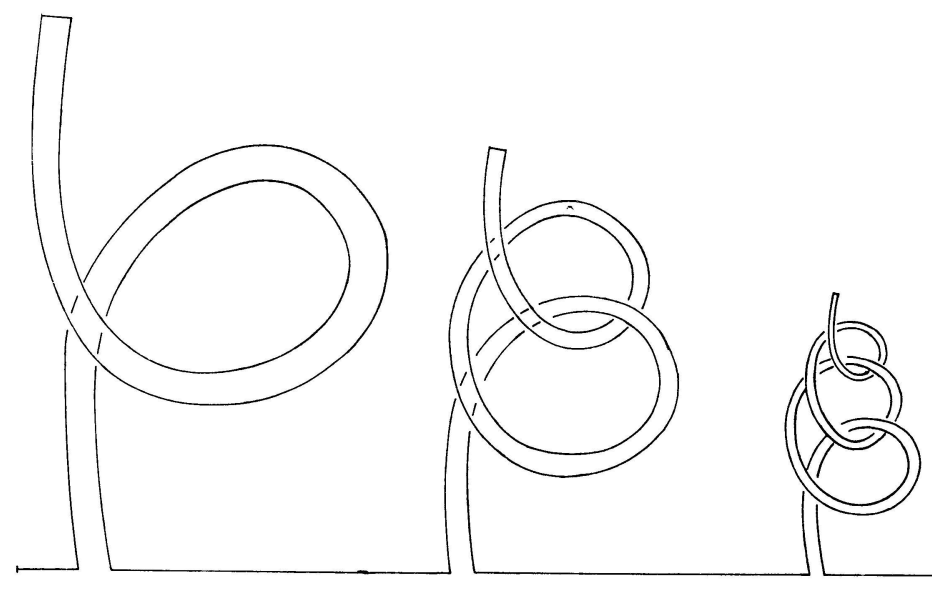

s. $X$

Figure 3

$g_{n}^{\prime \prime}$ we can define Lipschitz embeddings $F_{n}$ : cl $U_{1}(0) \rightarrow R^{3}$ with $F_{n}\left(I^{1}\right)=f_{n}\left(I^{1}\right)=$ $g_{n}^{\prime} \circ g \circ g_{n}^{\prime \prime}\left(I^{1}\right)$ if $B_{n} \subset U$. We can assume that there is $L^{\prime}>1$ such that each $F_{n}$ is an $L^{\prime}$-embedding. Therefore $\left\{F_{n}: B_{n} \subset U\right\}$ is a normal family, and there is a subsequence $F_{n(1)}, F_{n(2)}, \ldots$ such that there is a uniform $\operatorname{limit}_{\lim _{i \rightarrow \infty}} F_{n(i)}=F^{\prime}$. But we must have $F^{\prime}\left(I^{1}\right)=f\left(I^{1}\right)=J$, since $F_{n(i)}\left(I^{1}\right)=f_{n(i)}\left(I^{1}\right)$. This implies that $J$ is locally Lipschitz flat at all interior points. This is impossible since $J$ is not even locally topologically flat at the bad point of Fig. 2 . 
A succinct description of the situation is that $J_{0}$ contains an infinitesimal FoxArtin arc at $X$ which is invisible in a low-power TOP microscope but becomes visible in a high-resolution LIP microscope.

15. We can apply the same principle to construct a Lipschitz embedding of the sphere $S^{2}$ into $R^{3}$ which is topologically flat but not locally Lipschitz flat. First we take a Fox-Artin arc (i.e. the first half of the arc in Fig. 2) and fatten it in such a way that we obtain a tube converging to the bad point. In this manner we get a Lipschitz embedding $f: S^{2} \cap R_{+}^{3} \rightarrow R^{3}$ which is not locally topologically flat at $(0,0,1)$. Again, by cutting the tube a little before the bad point and by pasting a disk in place of it, we obtain a sequence $f_{n}: S^{2} \cap R_{+}^{3} \rightarrow R^{3}, n>0$, of embeddings such that each $f_{n}$ is topologically flat and that $\lim _{n \rightarrow \infty} f_{n}=f$. In addition, we may assume that $f$ and the $f_{n}$ 's are $L$-embeddings for some $L>1$. Now, we consider the sphere $S^{2} \subset R^{3}$. We remove from $S^{2}$ a disjoint family $E_{i}, i \geqq 1$, of disks converging towards a point $X$. In place of the disks $E_{i}$ we attach deformed disks similar to $f_{i}\left(S^{2} \cap R_{+}^{3}\right)$. We can do this in such a way that we obtain a Lipschitz embedding of $S^{2}$ into $R^{3}$ which is topologically flat but not locally Lipschitz flat at the point $X$. Details are as above.

16. These counter-examples show that topological flatness does not imply Lipschitz flatness. We now propose an additional condition and conjecture that this condition would guarantee Lipschitz flatness.

Let $f: I^{n}=I^{1} \times \ldots \times I^{1} \rightarrow R^{m}$ be an $L$-embedding. Let $x \in$ int $I^{n}$ and let $r>0$ satisfy $U_{r}(x) \subset I^{n}$. Define $f_{r x}: U_{1}(0) \rightarrow R^{m}$ by

$$
f_{r x}(y)=f(x)+(f(x+r y)-f(x)) / r
$$

for $y \in U_{1}(0)$. Then each $f_{r x}$ is an $L$-embedding.

Let $U \subset$ int $I^{n}$ be open and let

$$
\mathscr{L}_{f}(U)=\left\{f_{r x}: x \in U \text { and } U_{r}(x) \subset U\right\}
$$

be the set of Lipschitz germs of $f$ in $U$. Now we can state the following

Conjecture 1. The map $f$ is locally Lipschitz flat at $x \in$ int $I^{n}$ if and only if $x$ has a neighbourhood $U$ in int $I^{n}$ such that $\mathrm{cl} \mathscr{L}_{f}(U)$ contains only topologically flat germs.

In this conjecture closure is taken in the topology of uniform convergence of maps $U_{1}(0) \rightarrow R^{m}$. Then each element of $\mathrm{cl} \mathscr{L}_{f}(U)$ is an $L$-embedding, but the construction of the counter-examples in the preceding sections was based on the fact that elements of $\mathrm{cl} \mathscr{L}_{f}(U)$ need not be topologically flat even if elements of $\mathscr{L}_{f}(U)$ are. If $f$ is locally Lipschitz flat at $x$, then $x$ has such a neighbourhood $U$. Note that if $n=1$ and $m=2$, the assumptions of the conjecture are always satisfied, since there are only topologically flat embeddings of arcs into $R^{2}$.

Naturally, we can also formulate this conjecture for boundary points $x \in \partial I^{n}$. Now $\mathscr{L}_{f}(U)$ contains also maps $U_{1}(0) \cap R_{+}^{n} \rightarrow R^{m}$ and, if $x$ is a corner point, other 
kinds of maps, too. With this modification, it is expected that the above conjecture is true also for boundary points. Since often local flatness implies global flatness (cf. Gauld-Väisälä [4]), the global form of the conjecture is that every point has such a neighbourhood $U$.

If $n=1$ or $n=2$ and $m=3$, it seems likely that the conjecture can be proved like the results of this paper, only with much more labour.

17. The quasiconformal case. We constructed in Sections 14 and 15 an arc and a sphere in $R^{3}$ that are topologically flat but not locally Lipschitz flat. Moreover, they are not even locally quasiconformally flat. This can be shown by a normal family argument as in Section 14. Note that, if the map $F: U_{\lambda}(0) \rightarrow R^{3}$ of Section 14 were quasiconformal, then the maps $F_{n}$ can be assumed for large $n$ to be of the form $F_{n}=F_{n}^{\prime} \mid \mathrm{cl} U_{1}(0)$, where the maps $F_{n}^{\prime}$ are $K$-quasiconformal embeddings $U_{2}(0) \rightarrow R^{3}$ for some $K \geqq 1$. Since $F_{n}^{\prime}\left(I^{1}\right)=f_{n}\left(I^{1}\right)=J_{n}$, we have $F_{n}^{\prime}(\{-1,1\})=\{a, b\}$, where $a$ and $b$ are the endpoints of $J$. By [13, 19.4 (1)] (set $\left.a_{f}=\infty\right)$ and [13, 20.5] $\left\{F_{n}^{\prime}: B_{n} \subset U\right\}$ is a normal family. Therefore there are a subsequence $F_{n(1)}^{\prime}, F_{n(2)}^{\prime}, \ldots$ and a map $F^{\prime}: U_{2}(0) \rightarrow R^{3}$ such that $F_{n(i)}^{\prime} \rightarrow F^{\prime}$ uniformly on compact subsets. Since $\{a, b\} \subset \operatorname{im} F^{\prime}, F^{\prime}$ cannot be a constant. Then by [13, 21.1 and 37.4] $F^{\prime}$ must be a quasiconformal homeomorphism. This would imply again that the arc $J$ of Fig. 2 is locally quasiconformally flat at all points, which is impossible.

Conjecture 1 in the preceding section can be formulated also in the context of quasiconformal mappings. Let $f: R^{n} \rightarrow R^{m}$ be an embedding and let

$$
\begin{aligned}
\mathscr{K}_{f}= & \left\{\alpha f \beta: \alpha \text { a similarity of } R^{m}, \beta \text { a similarity of } R^{n},\right. \\
& \alpha f \beta(0)=0, \text { and } \alpha f \beta(1,0, \ldots, 0)=(1,0, \ldots, 0)\}
\end{aligned}
$$

be the set of quasiconformal germs of $f$. Now we make

Conjecture 2. There is a quasiconformal homeomorphism $F$ of $R^{m}$ with $F \mid R^{n}=f$ if and only if $\mathrm{cl} \mathscr{K}_{f}$ is compact and contains only topologically flat germs.

The closure is in the set of all embeddings $R^{n} \rightarrow R^{m}$ in the topology of uniform convergence on compact sets. If $f$ is of this form it is easy to see that the conditions of Conjecture 2 are satisfied. Also, it is possible to give a local form of the conjecture, as in the preceding section.

Note that one can characterize quasisymmetric and quasiconformal self-maps of $R^{n}, n \geqq 1$, by aid of compact families of mappings as we have done above; cf. Beurling-Ahlfors [2, Chapter 2] and Gehring [5, Section 32].

One can also define that an embedding $f: R^{n} \rightarrow R^{m}$ is quasisymmetric if there is $H \geqq 1$ such that if $x, y, z \in R^{n},\|x-z\| \leqq\|y-z\|$, then $\|f(x)-f(z)\| \leqq H\|f(y)-f(z)\|$. These mappings have compactness properties similar to the corresponding properties of quasiconformal mappings; cf. [12]. One can show ([12]) that an embedding $f: R^{n} \rightarrow R^{m}$ is quasisymmetric if and only if the family of quasiconformal germs of $f$ is contained in a compact family of embeddings. 
We could have adapted the abcve proof with minor modifications to the quasiconformal case, i.e., we could have given the necessary and sufficient conditions for the existence of a quasiconformal homeomorphism of $R^{2}$ extending a given embedding of the arc $I^{1}$ or the sphere $S$ into $R^{2}$. This could have been done, for instance, by aid of the notion of a quasisymmetric embedding given above. However, in view of the results of Ahlfors [1], Rickman [9, 10] and Reed [8], this is unnecessary.

In the quasiconformal case we can also give an absolute version of Conjecture 1. Let $\mathscr{C}^{m}$ be the family of non-empty closed subsets of $R^{m}$. If $X, Y \in \mathscr{C}^{m}$ let, when $d_{s}$ is the spherical metric of $R^{m}$,

$$
\varrho(X, Y)=\sup \left\{d_{s}(x, Y), d_{s}(X, y): x \in X, y \in Y\right\}
$$

Then $\varrho$ is a metric in $\mathscr{C}^{m}$ and we topologize $\mathscr{C}^{m}$ by means of this metric. For $C \in \mathscr{C}^{m}$ let $\mathscr{F}_{C}=\left\{\alpha(C): \alpha\right.$ a similarity of $R^{m}$ and $\left.\{0,1\} \subset \alpha(C)\right\} \subset \mathscr{C}^{m}$. Now we can formulate

Conjecture 3. Let $C \subset R^{m}$ be a subset such that $C \cup\{\infty\}$ is homeomorphic to the $n$-sphere $S^{n}, n \leqq m$. Then $C=f\left(R^{n}\right)$ for some quasiconformal homeomorphism fof $R^{m}$ if and only if $\mathrm{cl} \mathscr{F}_{C}$ is compact and every element of $\mathrm{cl} \mathscr{F}_{C}$ is of the form $g\left(R^{n}\right)$ for some homeomorphism $g$ of $R^{m}$.

It is easy to see that if $C=f\left(R^{n}\right), f$ a quasiconformal homeomorphism of $R^{m}$, $\mathscr{F}_{C}$ has the properties mentioned in the above conjecture.

\section{References}

[1] Ahlfors, L.: Quasiconformal reflections. - Acta Math. 109, 1963, 291-301.

[2] Beurling, A., and L. Ahlfors: The boundary correspondence under quasiconformal mappings. - Acta Math. 96, 1956, 125-142.

[3] Carleson, L.: The extension problem for quasiconformal mappings. - Contributions to Analysis, edited by L. V. Ahlfors et. al., Academic Press, New York-London, 1974, 39-47.

[4] GAULD, D. B., and J. VÄISÄLÄ: Lipschitz and quasiconformal flattening of spheres and cells. Ann. Acad. Sci. Fenn. Ser. A I 4, 1978/1979, 371-382.

[5] Gehring, F. W.: Rings and quasiconformal mappings in space. - Trans. Amer. Math. Soc. 103, 1962, 353-393.

[6] Luukkainen, J., and J. VÄisÄLÄ: Elements of Lipschitz topology. - Ann. Acad. Sci. Fenn. Ser. A I 3, 1977, 85-122.

[7] Moise, E. E.: Geometric topology in dimensions 2 and 3. - Springer-Verlag, New York-Heidelberg-Berlin, 1977.

[8] REED, T. J.: On the boundary correspondence of quasiconformal mappings of domains bounded by quasicircles. - Pacific J. Math. 28, 1969, 653-661.

[9] Rickman, S.: Characterization of quasiconformal arcs. - Ann. Acad. Sci. Fenn. Ser A I 395, 1966, 1-30. 
[10] Rickman, S.: Quasiconformally equivalent curves. - Duke Math. J. 36, 1969, 387-400.

[11] Tukia, P.: Lipschitz approximation of homeomorphisms. - Ann. Acad. Sci. Fenn. Ser. A I 4, 1978/1979, 137-144.

[12] TukIA, P., and J. VÄIsÄLÄ: Quasisymmetric embeddings of metric spaces. - Ann. Acad. Sci. Fenn. Ser. A I 5, 1980, 97-114.

[13] VÄISÄLÄ, J.: Lectures on $n$-dimensional quasiconformal mappings. - Lecture Notes in Mathematics 229, Springer-Verlag, Berlin-Heidelberg-New York, 1971.

[14] VÄISÄL Ä, J.: Piecewise linear approximation of lipeomorphisms. - Ann. Acad. Sci. Fenn. Ser. A I 3, 1977, 377-383.

University of Helsinki

Department of Mathematics

SF-00100 Helsinki 10

Finland

Received 2 February 1979

Revision received 15 August 1979 